Неклассическая логика

Non-classical Logic

Л.Ю. ДЕвяткин

\title{
О выразительных возможностях максимально паранепротиворечивых и максимально параполных четырехзначных расширений FDE
}

\author{
Леонид Юрьевич Девяткин \\ Институт философии РАН. \\ Российская Федерация, 109240, г. Москва, ул. Гончарная, д. 12, стр. 1. \\ E-mail: deviatkin@iph.ras.ru
}

Аннотация: Статья посвящена замкнутым классам функций четырехзначной логики, которые могут быть порождены системами элементарных операций характеристических матриц для таких языковых расширений логики FDE, которые являются одновременно максимально паранепротиворечивыми и максимально параполными.

Мы начинаем с представления необходимых и достаточных условий, которым должны отвечать четырехзначные языковые расширения FDE, чтобы быть максимально паранепротиворечивыми и максимально параполными. В обоих случаях критерии максимальности связаны с наличием в матрице рассматриваемого расширения операторов определенного рода, из-за которых это расширение не является подлогикой трехзначного языкового расширения логики Асеньо-Приста $\mathbf{L P}-$ в случае паранепротиворечивости и логики Клини $\mathbf{K}_{\mathbf{3}}-$ в случае параполноты.

Далее, опираясь на теорему Бэйкера-Пиксли, мы даем описание такого множества из 5 одноместных и 20 двухместных предикатов, что любой замкнутый класс функций, порожденный системой элементарных операций четырехзначной характеристической матрицы языкового расширения FDE, есть класс функций, сохраняющих одно из подмножеств данного множества. Это дает простой алгоритм сравнения выразительных возможностей любых произвольно взятых четырехзначных языковых расширений FDE.

Кроме того, принимая во внимание, что в приведенное множество предикатов включаются все предикаты, описывающие предполные классы функций четырехзначной логики, которые сохраняются операциями характеристической матрицы FDE, мы приводим критерии функциональной полноты и предполноты для множества всех операций любой четырехзначной матрицы, характеризующей языковое расширение FDE.

Наконец, используя критерии максимальной паранепротиворечивости и параполноты, а также список предикатов для расширений FDE, представленные в статье, мы идентифицируем все 14 множеств предикатов, описывающих замкнутые классы, которые соответствуют четырехзначным характеристическим матрицам максимально паранепротиворечивых и максимально параполных расширений FDE.

(C) Девяткин Л.Ю. 
Ключевые слова: четырехзначные логики, паранепротиворечивость, параполнота, замкнутые классы функций, замкнутые классы предикатов

Для цитирования: Девяткин Л.Ю. О выразительных возможностях максимально паранепротиворечивых и максимально параполных четырехзначных расширений FDE // Логические исследования / Logical Investigations. 2021. Т. 27. № 2. С. 66-92. DOI: 10.21146/2074-1472-2021-27-2-66-92

\section{Введение}

Настоящая работа затрагивает две сферы современных исследований в области неклассических логик. Первая из них - это изучение выразительных возможностей языков различных многозначных логик. Вторая паранепротиворечивые и параполные логики, в частности, проблема поиска критериев максимальности для паранепротиворечивости и параполноты. В основе методологии работы лежит рассмотрение выразительных возможностей логических матриц с точки зрения замкнутых классов функций, порождаемых их системами элементарных операций.

Уже в конце 40-х годов прошлого столетия исследователями многозначных логик ставились вопросы о выразительных возможностях их языков они касались как критериев определимости тех или иных операций в отдельно взятой матрице, так и подобных критериев для определимости операций одной матрицы в другой [Łoś, 1949, Ch. III]. Особенную актуальность эти вопросы приобретают, когда речь идет о матрице для логики FDE, предложенной Н.Д. Белнапом в [Belnap, 1977]. Эта актуальность продиктована тем, что популярным подходом к построению четырехзначных логик является пополнение матрицы FDE новыми операциями. Обзоры многочисленных логик, полученных таким образом, можно найти в Omori, Wansing, 2017] и [Petrukhin, Shangin, 2019]. Анализу и классификации расширений FDE с точки зрения выразительных возможностей их языков специально посвящены работы Arieli, Avron, 1998; Avron, 1999, Omori, Sano, 2015; Karpenko, 2017; Přenosil, 2021]. Наша работа лежит в русле данных исследований, дополняя уже известные результаты. При этом в фокусе внимания находятся расширения FDE, принадлежащие к числу паранепротиворечивых и параполных логик.

Паранепротиворечивые логики - это логики, в которых противоречия не ведут к тривиальности; параполные логики - это логики, в которых некоторая формула и ее отрицание могут быть одновременно ложны ${ }^{1}$. Вопросы паранепротиворечивости, связанные с расширениями FDE, затрагиваются в (Omori, Sano, 2014 и Arieli, Avron, 2017.

\footnotetext{
${ }^{1}$ Более строгие формальные определения будут даны ниже.
} 
Важную роль в исследованиях паранепротиворечивых логик играет понятие максимальности. В основе этого понятия лежит неформальный принцип, принадлежащий Н. Да Коста: полезная паранепротиворечивая логика должна содержать наибольший фрагмент классической логики, не влекущий потери паранепротиворечивости [Da Costa, 1974. Kaк указывают авторы Arieli et al., 2011, принятая в литературе интерпретация этого принципа состоит в том, что добавление любого фрагмента классической логики, который уже не содержится в рассматриваемой паранепротиворечивой логике, превращает эту логику в классическую. Если логика обладает этим свойством, ее называют максимально паранепротиворечивой относительно классической логики. Кроме того, в процитированной статье авторы предлагают следующее обобщение: логика является максимально паранепротиворечивой в абсолютном смьсле, если она паранепротиворечива, однако ни одна ее надлогика не является таковой. В нашей работе рассматривается максимальная противоречивость именно в абсолютном смысле. Аналогичным образом определяется понятие максимальной параполноты.

Число возможных четырехзначных расширений FDE бесконечно, поскольку бесконечно число алгебраических функций на четырехэлементном множестве. В то же время, расширения, различающиеся наборами элементарных операций, могут при этом совпадать по выразительным возможностям. Например, в Omori, Sano, 2015 демонстрируется «выразительная» эквивалентность для четырех расширений с попарно различными наборами элементарных операций. Поэтому при исследовании выразительных возможностей языков многозначных логик имеет смысл рассматривать не наборы их элементарных операций, а замкнутые классы функций, соответствующие множествам всех операций, определимых в рассматриваемых логиках. Примеры такого подхода можно найти в Adams, Dziobiak, 1994; Přenosil, 2021; Tomova, 2021. В первой работе найдены замкнутые классы функций, соответствующие всем расширениям $\mathbf{K}_{\mathbf{3}}$, сильной логики Клини ${ }^{2}$. Для описания этих классов используется предикатный подход, то есть каждый класс определяется как класс всех функций, сохраняющих некое множество предикатов. Во второй работе описаны замкнутые классы, соответствующие отдельным расширениям FDE, однако эти классы определяются на основе их порождающих систем, а не предикатов. В третьей работе рассматриваются замкнутые классы, порожденные некоторыми четырехзначными паранепротиворечивыми и параполными логиками. Изложенные ниже результаты сочетают черты этих работ: мы даем описа-

\footnotetext{
${ }^{2}$ Отметим, что логика $\mathbf{K}_{\mathbf{3}}$ связана с FDE существенным для настоящей работы образом. Мы вернемся к этой связи позже.
} 
ния замкнутых классов функций для паранепротиворечивых и параполных четырехзначных расширений FDE определенного типа, применяя предикатный подход.

Дальнейшее изложение материала построено следующим образом. Первый раздел посвящен необходимым и достаточным условиям того, чтобы логика, полученная добавлением к FDE новых операций, являлась максимально паранепротиворечивой (Теорема 1) или максимально параполной (Теорема 2). Второй раздел содержит два основных результата. Во-первых, даются необходимые и достаточные условия функциональной полноты и предполноты для произвольных четырехзначных расширений FDE (Теорема 3). Во-вторых, дается полный список замкнутых классов, соответствующих четырехзначным расширениям FDE, которые являются одновременно максимально паранепротиворечивыми и максимально параполными (Теорема 4). В заключении рассмотрены возможные приложения полученных результатов, а также направления дальнейших исследований.

\section{1. Критерии максимальной паранепротиворечивости и параполноты в четырехзначных языковых расширениях FDE}

В этом разделе мы показываем, что четырехзначное языковое расширение FDE является максимально паранепротиворечивым, е.т.е. его матрица содержит операцию, которая дает значение $\mathbf{n}$ на некотором наборе, все элементы которого принадлежат множеству $\{\mathbf{t}, \mathbf{b}, \mathbf{f}\}$ (Теорема 1), и что оно является максимально параполным е.т.е. его матрица содержит операцию, которая дает значение b на некотором наборе, все элементы которого принадлежат множеству $\{\mathbf{t}, \mathbf{n}, \mathbf{f}\}$ (Теорема 2). Структура раздела такова. Сперва мы даем определения необходимых понятий, таких как пропозициональный язык, отношение следования, пропозициональная логика, логическая матрица, многозначная логика, паранепротиворечивость, параполнота, максимальность паранепротиворечивости и параполноты. Отдельно отметим, что при определении базовых понятий используется определение следования с множественными заключениями из Shoesmith, Smiley, 1978, p. 29]. Причина этого в том, что оно наиболее удобно для работы с понятиями паранепротиворечивости и параполноты [Marcos, 2005]. После основных определений мы останавливаемся на четырехзначной матрице для FDE и демонстрируем, что логика FDE не является ни максимально паранепротиворечивой, ни максимально параполной. Далее следует описание произвольного четырехзначного языкового расширения FDE и его характеристической матрицы. Формулировки основных теорем и их доказательства завершают раздел. 
Называем пропозичиональным языком $\mathcal{L}=\left\langle F m(\mathcal{L}), \S_{1}, \ldots, \S_{k}\right\rangle$ алгебру, свободно порожденную счетным множеством пропозициональных переменных $\operatorname{Var}(\mathcal{L})$, которая свободна в классе всех алгебр аналогичной сигнатуры. Говорим, что $\vdash$ является отношением следования на $\mathcal{L}$, когда следующие условия выполняются для всех $X, Y, S \subseteq F m(\mathcal{L})$ :

- если $X \cap Y \neq \varnothing$, то $X \vdash Y$ (рефлексивность);

- если $X^{\prime} \vdash Y^{\prime}$, где $X^{\prime} \subseteq X$ и $Y^{\prime} \subseteq Y$, то $X \vdash Y$ (монотонность);

- если для всех $Z \subseteq S$ имеет место $X \cup Z \vdash Y \cup(S \backslash Z)$, то $X \vdash Y$ (сечение).

Когда вдобавок $X \vdash Y \Longrightarrow \varepsilon(X) \vdash \varepsilon(Y)$ для каждого эндоморфизма $\varepsilon$ языка $\mathcal{L}$, называем отношение следования $\vdash$ структурным. Если $\mathcal{L}-$ пропозициональный язык и $\vdash-$ структурное отношение следования на $\mathcal{L}$, говорим, что $\mathbf{L}=\langle\mathcal{L}, \vdash\rangle-$ пропозициональная логика.

Пусть $\mathbf{L}=\langle\mathcal{L}, \vdash\rangle$ и $\mathbf{L}^{*}=\left\langle\mathcal{L}, \vdash^{*}\right\rangle-$ пропозициональные логики в одном и том же языке. Если $X \vdash Y \Longrightarrow X \vdash^{*} Y$ для всех $X, Y \subseteq F m(\mathcal{L})$, называем $\mathbf{L}^{*}$ дедуктивным расширением $\mathbf{L}$. Если, кроме того, найдутся такие $X^{\prime}, Y^{\prime} \subseteq F m(\mathcal{L})$, что $X^{\prime} \vdash^{*} Y^{\prime}$ и $X^{\prime} \nvdash Y^{\prime}$, говорим, что $\mathbf{L}^{*}-$ собственное дедуктивное расширение $\mathbf{L}$, а $\mathbf{L}-$ подлогика $\mathbf{L}^{*}$.

Пусть $\mathbf{L}_{1}=\left\langle\mathcal{L}_{1}, \vdash_{1}\right\rangle$ и $\mathbf{L}_{2}=\left\langle\mathcal{L}_{2}, \vdash_{2}\right\rangle-$ пропозициональные логики, где $\operatorname{Var}\left(\mathcal{L}_{1}\right)=\operatorname{Var}\left(\mathcal{L}_{2}\right)$ и $F m\left(\mathcal{L}_{1}\right) \subseteq F m\left(\mathcal{L}_{2}\right)$. Если $X \vdash_{1} Y \Longleftrightarrow X \vdash_{2} Y$ для всех $X, Y \subseteq F m\left(\mathcal{L}_{1}\right)$, говорим, что $\mathbf{L}_{2}-$ языковое расширение $\mathbf{L}_{1}$.

Называем логику $\mathbf{L}=\langle\mathcal{L}, \vdash\rangle$ паранепротиворечивой (относительно отрицания $\left.^{3} \neg\right)$, если $\alpha, \neg \alpha \nvdash \beta$ для некоторых $\alpha, \beta \in F m(\mathcal{L})$. Называем логику $\mathbf{L}=\langle\mathcal{L}, \vdash\rangle$ параполной (относительно отрицания $\neg$ ), если $\alpha \nvdash \beta, \neg \beta$ для некоторых $\alpha, \beta \in F m(\mathcal{L})$. Если $\mathbf{L}$ паранепротиворечива (параполна), но никакое ее собственное дедуктивное расширение не является таковым, называем $\mathbf{L}$ максимально паранепротиворечивой (параполной) в абсолютном смысле.

Логическая матрица - это структура $\mathcal{M}=\left\langle A, f_{1}, \ldots, f_{k}, D\right\rangle$, где $\mathcal{A}=\left\langle A, f_{1}, \ldots, f_{k}\right\rangle$ - алгебра, а $D$ - подмножество $A$. Функции $f_{1}, \ldots, f_{k}$ называем элементарными операциями $\mathcal{M}$. Если $\mathcal{L}=\left\langle F m(\mathcal{L}), \S_{1}, \ldots, \S_{k}\right\rangle$ и $\mathcal{A}=\left\langle A, f_{\S_{1}}, \ldots, f_{\S_{k}}\right\rangle$, где местность $\S_{i}$ совпадает с местностью $f_{\S_{i}}$ для каждого $1 \geq i \geq k$, говорим, что $\mathcal{M}$ - матрица для $\mathcal{L}$. Когда $\mathcal{M}$ - матрица для $\mathcal{L}$, гомоморфизм $h$ из $\mathcal{L}$ в $\mathcal{A}$ называем оценкой $\mathcal{L}$ в $\mathcal{M}$. Будем обозначать

\footnotetext{
${ }^{3}$ Вопрос о том, какими свойствами должна обладать унарная связка $\neg$ в общем случае, чтобы ее можно было считать отрицанием, выходит за рамки данной работы, поскольку в ней рассматриваются только паранепротиворечивость и параполнота относительно отрицания логики FDE, которое определяется на с. 71
} 
множество всех подобных оценок как $\operatorname{Val}(\mathcal{M})$. Определяем матричное отношение следования $C n(\mathcal{M})$ на $\mathcal{L}$, порожденное $\mathcal{M}$, следующим образом:

$$
C n(\mathcal{M})=\left\{\langle X, Y\rangle \mid \forall h_{\in \operatorname{Val}(\mathcal{M})}(h(X) \subseteq D \Longrightarrow h(Y) \cap D \neq \varnothing)\right\} .
$$

Если даны пропозициональная логика $\mathbf{L}=\langle\mathcal{L}, \vdash\rangle$ и матрица $\mathcal{M}$ для $\mathcal{L}$, говорим, что $\mathcal{M}$ является характеристической для $\mathbf{L}$, если $X \vdash Y \Longleftrightarrow\langle X, Y\rangle \in C n(\mathcal{M})$. Называем пропозициональную логику $\mathbf{L}=\langle\mathcal{L}, \vdash\rangle k$-значной, если существует $k$-значная матрица, которая является характеристической для $\mathbf{L}$.

Четырехзначная характеристическая матрица для FDE была дана Н.Д. Белнапом Belnap, 1977]. Она имеет вид $\mathcal{M}_{\mathbf{F D E}}=$ $\langle\{\mathbf{t}, \mathbf{b}, \mathbf{n}, \mathbf{f}\}, \wedge, \vee, \neg,\{\mathbf{t}, \mathbf{b}\}\rangle, \quad$ где элементарные операции определяются следующими таблицами ${ }^{4}$ :

\begin{tabular}{|c|c|c|c|c|c|c|c|c|c|c|c|}
\hline$\wedge$ & $\mathbf{t}$ & b & $\mathbf{n}$ & f & $\vee$ & t & b & $\mathbf{n}$ & f & $x$ & $\neg x$ \\
\hline $\mathbf{t}$ & $\mathrm{t}$ & b & $\mathbf{n}$ & f & $\mathbf{t}$ & $\mathrm{t}$ & $t$ & $\mathrm{t}$ & $\mathrm{t}$ & $\mathbf{t}$ & $\mathbf{f}$ \\
\hline b & b & b & f & $\mathbf{f}$ & $\mathbf{b}$ & $\mathbf{t}$ & b & $\mathbf{t}$ & b & b & $\mathbf{b}$ \\
\hline $\mathbf{n}$ & $\mathbf{n}$ & f & $\mathbf{n}$ & f & $\mathbf{n}$ & $t$ & $\mathbf{t}$ & $\mathbf{n}$ & $\mathbf{n}$ & $\mathbf{n}$ & $\mathbf{n}$ \\
\hline f & f & f & f & f & $\mathbf{f}$ & $\mathbf{t}$ & b & $\mathbf{n}$ & f & f & $\mathbf{t}$ \\
\hline
\end{tabular}

Обратим внимание, что в оригинальной формулировке FDE предполагает следование исключительно между парами индивидуальных формул вида $\alpha \vdash \beta$, а не между множествами формул ${ }^{5}$. В то же время табличные определения $\wedge$ и $\vee$ таковы, что для любых конечных множеств формул выполняется следующее:

$$
\begin{gathered}
\left\langle\left\{\alpha_{1}, \ldots, \alpha_{m}\right\},\left\{\beta_{1}, \ldots, \beta_{n}\right\}\right\rangle \in C n\left(\mathcal{M}_{\text {FDE }}\right) \Longleftrightarrow \\
\Longleftrightarrow\left\langle\left\{\alpha_{1} \wedge \cdots \wedge \alpha_{m}\right\},\left\{\beta_{1} \vee \cdots \vee \beta_{n}\right\}\right\rangle \in C n\left(\mathcal{M}_{\mathbf{F D E}}\right) .
\end{gathered}
$$

Это демонстрирует, что все результаты, представленные ниже в рамках выбранной нами системы базовых понятий, имеют место также для FDE в ее изначальном определении.

Известно, что логика FDE является одновременно паранепротиворечивой и параполной. В том, что $\langle\{p, \neg p\},\{q\}\rangle \notin C n\left(\mathcal{M}_{\mathbf{F D E}}\right)$ и $\langle\{p\},\{q \neg q\}\rangle \notin C n\left(\mathcal{M}_{\mathbf{F D E}}\right)$, нетрудно убедиться, посмотрев на табличное определение ᄀ. Однако FDE ни максимально паранепротиворечива, ни максимально параполна. Чтобы показать это, используем связь между $\mathbf{F D E}$ и $\mathbf{K}_{\mathbf{3}}$, о которой упоминали раньше, а также логикой парадокса $\mathbf{L P}$.

\footnotetext{
${ }^{4} \mathrm{~B}$ дальнейшем, злоупотребляя нотацией, будем использовать символы $\wedge, \vee, \neg$ как для обозначения пропозициональных связок, так и для обозначения соответствующих им матричных операторов.

${ }^{5}$ Вариант FDE со следованием, где посылки и заключения являются множествами формул, как в нашем случае, рассматривается в [Shapiro, 2017].
} 
Каждая из операций $\wedge, \vee, \neg$ является алгебраической функцией ${ }^{6}$ как на $\{\mathbf{t}, \mathbf{b}, \mathbf{f}\}$, так и на $\{\mathbf{t}, \mathbf{n}, \mathbf{f}\}$. Это означает, что $\mathcal{M}_{\mathbf{F D E}}$ имеет подматрицы ${ }^{7}$ с множествами-носителями $\{\mathbf{t}, \mathbf{b}, \mathbf{f}\}$ и на $\{\mathbf{t}, \mathbf{n}, \mathbf{f}\}$. Как отмечает $Г$. Прист, первая является характеристической матрицей для логики $\mathbf{L P}$, a вторая для логики $\mathbf{K}_{\mathbf{3}}$, и поэтому FDE является подлогикой $\mathbf{K}_{\mathbf{3}}$ и $\mathbf{L P}$ Priest, $2008, \S 8.4]$. При этом $\langle\{p, \neg p\},\{q\}\rangle \notin C n\left(\mathcal{M}_{\mathbf{L P}}\right),\langle\{p\},\{q, \neg q\}\rangle \in C n\left(\overline{\mathcal{M}}_{\mathbf{L P}}\right)$, $\langle\{p, \neg p\},\{q\}\rangle \in C n\left(\mathcal{M}_{\mathbf{K}_{3}}\right),\langle\{p\},\{q, \neg q\}\rangle \notin C n\left(\mathcal{M}_{\mathbf{K}_{3}}\right)$. Таким образом, LP паранепротиворечивое собственное дедуктивное расширение $\mathbf{F D E}, \mathbf{K}_{\mathbf{3}}-$ параполное собственное дедуктивное расширение FDE. Следовательно, FDE не является ни максимально паранепротиворечивой, ни максимально параполной.

Будем обозначать как $\mathbf{F D E}^{\mathbf{x}}$ произвольно взятое четырехзначное языковое расширение FDE. В силу введенных выше определений такое расширение имеет характеристическую матрицу вида $\mathcal{M}_{\mathbf{F D E}^{\mathbf{x}}}=\left\langle\{\mathbf{t}, \mathbf{b}, \mathbf{n}, \mathbf{f}\}, \wedge, \vee, \neg, f_{1}, \ldots, f_{k},\{\mathbf{t}, \mathbf{b}\}\right\rangle$, где $f_{1}, \ldots, f_{k}-$ функции, соответствующие новым связкам, добавленным к алфавиту FDE.

Теорема 1. Пусть $\mathbf{F D E}^{\mathbf{x}}$ - четырехзначное языковое расширение FDE. Логика $\mathbf{F D E}^{\mathbf{x}}$ является максимально паранепротиворечивой, е.т.е. ее характеристическая матрица $\mathcal{M}_{\mathbf{F D E}^{\mathbf{x}}}$ содержит такую элементарную операчию $f$, что $f\left(a_{1}, \ldots, a_{n}\right)=\mathbf{n}$ на некотором наборе $\left(a_{1}, \ldots, a_{n}\right) \in\{\mathbf{t}, \mathbf{b}, \mathbf{f}\}^{n}$.

Доказательство. Докажем достаточность. Пусть $\mathbf{F D E}^{\mathbf{x}+}-$ собственное дедуктивное расширение $\mathbf{F D E}^{\mathbf{x}}$. Тогда найдутся такие множества формул $X$ и $Y$, что $h^{*}(X) \subseteq\{\mathbf{t}, \mathbf{b}\}$ и $h^{*}(Y) \subseteq\{\mathbf{n}, \mathbf{f}\}$ для некоторой оценки $h^{*}$ в $\mathcal{M}_{\mathbf{F D E}}{ }^{\mathbf{x}}$, однако $X \vdash Y$ в $\mathbf{F D E}^{\mathbf{x}+}$. Пусть $\epsilon-$ подстановка, причем для каждой формулы $\gamma \in X$ и каждой формулы $\alpha \in Y$ верно, что $p_{0}, \neg p_{0} \vdash \epsilon(\gamma)$ и $p_{0}, \neg p_{0}, \epsilon(\alpha) \vdash q_{0}$ в $\mathbf{F} \mathbf{D E} \mathbf{E}^{\mathbf{x}}$. Поскольку $\mathbf{F} \mathbf{D E} \mathbf{E}^{\mathbf{x}+}-$ собственное дедуктивное расширение $\mathbf{F D E}^{\mathbf{x}}, p_{0}, \neg p_{0} \vdash \epsilon(\gamma)$ для всех $\gamma \in X$ и $p_{0}, \neg p_{0}, \epsilon(\alpha) \vdash q_{0}$ для всех $\alpha \in Y$ также в $\mathbf{F D E}^{\mathbf{x}+}$. Кроме того, в силу структурности $\epsilon(X) \vdash \epsilon(Y)$ в $\mathbf{F D E}^{\mathbf{x}+}$. (1) Из $p_{0}, \neg p_{0} \vdash \epsilon(\gamma)$ получаем $p_{0}, \neg p_{0}, Z \vdash(\epsilon(X) \cup \epsilon(Y)) \backslash Z, q_{0}$ для любого такого $Z \subseteq \epsilon(X) \cup \epsilon(Y)$, что $\epsilon(\gamma) \in(\epsilon(X) \cup \epsilon(Y)) \backslash Z$ для некоторой формулы $\gamma \in X$ (монотонность). (2) Из $p_{0}, \neg p_{0}, \epsilon(\alpha) \vdash q_{0}$ получаем $p_{0}, \neg p_{0}, Z \vdash(\epsilon(X) \cup \epsilon(Y)) \backslash Z, q_{0}$ для любого такого $Z \subseteq \epsilon(X) \cup \epsilon(Y)$, что $\epsilon(\alpha) \in Z$ для некоторой формулы $\alpha \in Y$ (монотонность). (3) Из $\epsilon(X) \vdash \epsilon(Y)$

\footnotetext{
${ }^{6}$ Алгебраической функцией на множестве $A$ называем такую функцию от $n$ переменных, которая дает значение из $A$ на каждом наборе из $A^{n}$.

${ }^{7}$ Называем $M=\left\langle A, f_{1}, \ldots, f_{k}, D\right\rangle$ подматрицей матрицы $N=\left\langle B, g_{1}, \ldots, g_{k}, E\right\rangle$, если $A \subseteq B, D \subseteq E$ и на каждом наборе $\left(a_{1}, \ldots, a_{n}\right) \in A^{n}$ для всех $i \in\{1, \ldots, k\}$ выполняется $f_{i}\left(a_{1}, \ldots, a_{n}\right)=g_{i}\left(a_{1}, \ldots, a_{n}\right)$.
} 
получаем $p_{0}, \neg p_{0}, Z \vdash(\epsilon(X) \cup \epsilon(Y)) \backslash Z, q_{0}$ для $Z=\epsilon(X)$, то есть единственного такого $Z \subseteq \epsilon(X) \cup \epsilon(Y)$, что $\epsilon(\gamma) \notin(\epsilon(X) \cup \epsilon(Y)) \backslash Z$ для всех $\gamma \in X$ и $\epsilon(\alpha) \notin Z$ для всех $\alpha \in Y$ (монотонность). Из (1)-(3) получаем, что для всех $Z \subseteq \epsilon(X) \cup \epsilon(Y)$ имеет место $p_{0}, \neg p_{0}, Z \vdash(\epsilon(X) \cup \epsilon(Y)) \backslash Z, q_{0}$. Таким образом, $p_{0}, \neg p_{0} \vdash q_{0}$ в $\mathbf{F} \mathbf{D E}^{\mathbf{x}+}$ (сечение). В силу последнего логика $\mathbf{F D E}{ }^{\mathbf{x}}$ не является паранепротиворечивой. Теперь покажем, что нужная подстановка $\epsilon$ всегда существует.

Случай 1 . В $\mathcal{M}_{\mathbf{F D E}^{\mathbf{x}}}$ содержится такая элементарная операция $g^{\prime}$, существенно зависящая от $n$ переменных, что $g^{\prime}(\mathbf{b}, \ldots, \mathbf{b}) \in\{\mathbf{t}, \mathbf{n}, \mathbf{f}\}$. Тогда в $\mathcal{M}_{\mathbf{F D E}}$ определима такая унарная операция $g$, что $g(\mathbf{b}) \in\{\mathbf{t}, \mathbf{n}, \mathbf{f}\}$. Всегда можно получить $g$ из $g^{\prime}$ отождествлением переменных. Это означает, что в $\mathcal{M}_{\mathbf{F D E}^{\mathbf{x}}}$ можно также определить такую операцию $g^{*}$, что $g^{*}(\mathbf{b})=\mathbf{n}$. Покажем это. Если $g(\mathbf{b})=\mathbf{n}$, утверждение тривиально. Допустим, что $g(\mathbf{b})=\mathbf{t}$. По условию $\mathcal{M}_{\mathbf{F D E}^{\mathbf{x}}}$ содержит такую операцию $f$, что $f\left(a_{1}, \ldots, a_{n}\right)=\mathbf{n}$ на некотором наборе $\left(a_{1}, \ldots, a_{n}\right) \in\{\mathbf{t}, \mathbf{b}, \mathbf{f}\}^{n}$. Тогда $g^{*}=f\left(\phi\left(x_{1}\right), \ldots, \phi\left(x_{n}\right)\right)$, где для всех $i \in\{1, \ldots, n\}$

$$
\phi\left(x_{i}\right)=\left\{\begin{array}{l}
g\left(x_{0}\right), \text { если } a_{i}=\mathbf{t}, \\
x_{0}, \text { если } a_{i}=\mathbf{b}, \\
\neg g\left(x_{0}\right), \text { если } a_{i}=\mathbf{f} .
\end{array}\right.
$$

Рассуждение для $g(\mathbf{b})=\mathbf{f}$ аналогично, достаточно поменять местами $g\left(x_{0}\right)$ и $\neg g\left(x_{0}\right)$ в определении $\phi$. Пусть формула $\delta(p)$ выражает функцию $g^{*}\left(x_{0}\right)$, то есть $h(\delta(p))=g^{*}(h(p))$ для любой оценки $h$ в $\mathcal{M}_{\mathbf{F D E}}$.

Обозначим как $\operatorname{Var}(X, Y)$ множество $\left\{p_{1}, \ldots, p_{k}\right\}$ всех переменных, входящих в формулы, принадлежащие $X$ или $Y$. Рассмотрим такую подстановку $\epsilon$, что для каждой переменной $p \in \operatorname{Var}(X, Y)$ выполняется приведенное ниже условие:

$$
\epsilon(p)=\left\{\begin{array}{l}
p_{0} \vee \delta\left(p_{0}\right), \text { если } h^{*}(p)=\mathbf{t}, \\
p_{0}, \text { если } h^{*}(p)=\mathbf{b}, \\
\delta\left(p_{0}\right), \text { если } h^{*}(p)=\mathbf{n}, \\
p_{0} \wedge \delta\left(p_{0}\right), \text { если } h^{*}(p)=\mathbf{f} .
\end{array}\right.
$$

Пусть $h\left(p_{0}\right)=\mathbf{b}$. Тогда $h\left(\delta\left(p_{0}\right)\right)=g^{*}\left(h\left(p_{0}\right)\right)=\mathbf{n} ; h\left(p_{0} \vee \delta\left(p_{0}\right)\right)=$ $h\left(p_{0}\right) \vee h\left(\delta\left(p_{0}\right)\right)=\mathbf{b} \vee \mathbf{n}=\mathbf{t} ; h\left(p_{0} \wedge \delta\left(p_{0}\right)\right)=h\left(p_{0}\right) \wedge h\left(\delta\left(p_{0}\right)\right)=\mathbf{b} \wedge \mathbf{n}=\mathbf{f}$. Поэтому для каждой оценки $h$ в $\mathcal{M}_{\mathbf{F D E x}}$ имеет место следующее: если $h\left(p_{0}\right)=\mathbf{b}$, то $h(\epsilon(\gamma))=h^{*}(\gamma) \in\{\mathbf{t}, \mathbf{b}\}$ для всех $\gamma \in X$ и $h(\epsilon(\alpha))=h^{*}(\alpha) \in\{\mathbf{n}, \mathbf{f}\}$ для всех $\alpha \in Y$. Это означает, что $p_{0}, \neg p_{0} \vdash \epsilon(\gamma)$ и $p_{0}, \neg p_{0}, \epsilon(\alpha) \vdash q_{0}$ в $\mathbf{F D E}^{\mathbf{x}}$ для всех $\gamma \in X$ и всех $\alpha \in Y$. 
Случай 2. Для каждой операции $g$ в $\mathcal{M}_{\mathbf{F D E}}$ верно, что $g(\mathbf{b}, \ldots, \mathbf{b})=\mathbf{b}$. Поскольку $\mathcal{M}_{\mathbf{F D E}}$ содержит такую операцию $f$, что $f\left(a_{1}, \ldots, a_{n}\right)=\mathbf{n}$ на некотором наборе $\left(a_{1}, \ldots, a_{n}\right) \in\{\mathbf{t}, \mathbf{b}, \mathbf{f}\}^{n}$, найдется такая формула $\beta$, не содержащая переменных из $\operatorname{Var}(X, Y)$, что для некоторой оценки $h^{\mathbf{n}}$ в $\mathcal{M}_{\mathbf{F D E}}$ выполняется следующее условие: $h^{\mathbf{n}}(\beta)=\mathbf{n}$ и $h^{\mathbf{n}}(r) \in\{\mathbf{t}, \mathbf{b}, \mathbf{f}\}$ для каждой $r \in \operatorname{Var}(\beta)$, где $\operatorname{Var}(\beta)$ есть множество $\left\{r_{1}, \ldots, r_{m}\right\}$ всех переменных, входящих в $\beta$.

Рассмотрим такую подстановку $\epsilon$, что для каждой переменной $r \in \operatorname{Var}(\beta)$ и каждой переменной $p \in \operatorname{Var}(X, Y)$ выполняются приведенные ниже условия:

$$
\begin{gathered}
\epsilon(r)=\left\{\begin{array}{l}
\left(p_{0} \vee q_{0}\right) \vee\left(\neg p_{0} \vee \neg q_{0}\right), \text { если } h^{\mathbf{n}}(r)=\mathbf{t}, \\
p_{0}, \text { если } h^{\mathbf{n}}(r)=\mathbf{b}, \\
\left(p_{0} \wedge q_{0}\right) \wedge\left(\neg p_{0} \wedge \neg q_{0}\right), \text { если } h^{\mathbf{n}}(r)=\mathbf{f} .
\end{array}\right. \\
\epsilon(p)=\left\{\begin{array}{l}
\left(p_{0} \vee q_{0}\right) \vee\left(\neg p_{0} \vee \neg q_{0}\right), \text { если } h^{*}(p)=\mathbf{t}, \\
p_{0}, \text { если } h^{*}(p)=\mathbf{b}, \\
\epsilon(\beta), \text { если } h^{*}(p)=\mathbf{n}, \\
\left(p_{0} \wedge q_{0}\right) \wedge\left(\neg p_{0} \wedge \neg q_{0}\right), \text { если } h^{*}(p)=\mathbf{f} .
\end{array}\right.
\end{gathered}
$$

Пусть $h\left(p_{0}\right)=\mathbf{b}$. Если $h\left(q_{0}\right) \in\{\mathbf{t}, \mathbf{n}\}$, то $h\left(p_{0} \vee q_{0}\right)=\mathbf{t}$. Если $h\left(q_{0}\right)=\mathbf{f}$, то $h\left(\neg p_{0} \vee \neg q_{0}\right)=\mathbf{t}$. Таким образом, если $h\left(p_{0}\right)=\mathbf{b}$ и $h\left(q_{0}\right) \in\{\mathbf{t}, \mathbf{n}, \mathbf{f}\}$, то $h\left(\left(p_{0} \vee q_{0}\right) \vee\left(\neg p_{0} \vee \neg q_{0}\right)\right)=\mathbf{t}$. Далее, если $h\left(q_{0}\right) \in\{\mathbf{n}, \mathbf{f}\}$, то $h\left(p_{0} \wedge q_{0}\right)=\mathbf{f}$. Если же $h\left(q_{0}\right)=\mathbf{t}$, то $h\left(\neg p_{0} \wedge \neg q_{0}\right)=\mathbf{f}$. Таким образом, если $h\left(p_{0}\right)=\mathbf{b}$ и $h\left(q_{0}\right) \in\{\mathbf{t}, \mathbf{n}, \mathbf{f}\}$, то $h\left(\left(p_{0} \wedge q_{0}\right) \wedge\left(\neg p_{0} \wedge \neg q_{0}\right)\right)=\mathbf{f}$. Следовательно, если $h\left(p_{0}\right)=\mathbf{b}$ и $h\left(q_{0}\right) \in\{\mathbf{t}, \mathbf{n}, \mathbf{f}\}$, то $h(\epsilon(\beta))=h^{\mathbf{n}}(\beta)=\mathbf{n}$. В свою очередь, это означает, что для каждой оценки $h$ в $\mathcal{M}_{\mathbf{F D E}^{\mathbf{x}}}$ имеет место следующее: если $h\left(p_{0}\right)=\mathbf{b}$ и $h\left(q_{0}\right) \in\{\mathbf{t}, \mathbf{n}, \mathbf{f}\}$, то $h(\epsilon(\gamma))=h^{*}(\gamma) \in\{\mathbf{t}, \mathbf{b}\}$ для всех $\gamma \in X$ и $h(\epsilon(\alpha))=h^{*}(\alpha) \in\{\mathbf{n}, \mathbf{f}\}$ для всех $\alpha \in Y$. Кроме того, поскольку для каждой операции $g$ в $\mathcal{M}_{\mathbf{F D E}^{\mathbf{x}}}$ верно, что $g(\mathbf{b}, \ldots, \mathbf{b})=\mathbf{b}$, если $h\left(p_{0}\right)=h\left(q_{0}\right)=\mathbf{b}$, то $h(\epsilon(\gamma))=h(\epsilon(\alpha))=\mathbf{b}$ для всех $\gamma \in X$ и всех $\alpha \in Y$. Таким образом, снова $p_{0}, \neg p_{0} \vdash \epsilon(\gamma)$ и $p_{0}, \neg p_{0}, \epsilon(\alpha) \vdash q_{0}$ в $\mathbf{F D E}^{\mathbf{x}}$ для всех $\gamma \in X$ и всех $\alpha \in Y$. Достаточность доказана.

Теперь докажем необходимость. Допустим, что для каждой операции $f$ в $\mathcal{M}_{\mathbf{F D E}^{\mathbf{x}}}$ верно, что $f\left(a_{1}, \ldots, a_{n}\right) \in\{\mathbf{t}, \mathbf{b}, \mathbf{f}\}$ для каждого набора $\left(a_{1}, \ldots, a_{n}\right) \in\{\mathbf{t}, \mathbf{b}, \mathbf{f}\}^{n}$. В этом случае $\mathcal{M}_{\mathbf{F D E}^{\mathbf{x}}}$ содержит подматрицу вида $\mathcal{M}_{\mathbf{L P}}=\left\langle\{\mathbf{t}, \mathbf{b}, \mathbf{f}\}, \wedge, \vee, \neg, f_{1}, \ldots, f_{k},\{\mathbf{t}, \mathbf{b}\}\right\rangle$. Имеют место следующие факты: если $\langle X, Y\rangle \in C n\left(\mathcal{M}_{\mathbf{F D E}}\right)$, то $\langle X, Y\rangle \in C n\left(\mathcal{M}_{\mathbf{L P}} \mathbf{x}\right)$; $\langle\{p, \neg p\},\{q, \neg q\}\rangle \notin C n\left(\mathcal{M}_{\mathbf{F D E}^{\mathbf{x}}}\right)$, но $\langle\{p, \neg p\},\{q, \neg q\}\rangle \in C n\left(\mathcal{M}_{\mathbf{L P}} \mathbf{x}\right) ;$ $\langle\{p, \neg p\},\{q\}\rangle \notin C n\left(\mathcal{M}_{\mathbf{L P}} \mathbf{x}\right)$. Следовательно, трехзначная логика $\mathbf{L P}^{\mathbf{x}}$, для 
которой матрица $\mathcal{M}_{\mathbf{L P}}$ я является характеристической, - это паранепротиворечивое собственное дедуктивное расширение $\mathbf{F D E}^{\mathbf{x}}$. Таким образом, $\mathbf{F D E}^{\mathbf{x}}$ не максимально паранепротиворечива.

Теорема 2. Пусть $\mathbf{F D E}^{\mathbf{x}}$ - четырехзначное языковое расширение $\mathbf{F D E}$. Логика $\mathbf{F D E}^{\mathbf{x}}$ является максимально параполной, е.т.е. ее характеристическая матрица $\mathcal{M}_{\mathbf{F D E}^{x}}$ содержсит такую элементарную операцию $f$, что $f\left(a_{1}, \ldots, a_{n}\right)=\mathbf{b}$ на некотором наборе $\left(a_{1}, \ldots, a_{n}\right) \in\{\mathbf{t}, \mathbf{n}, \mathbf{f}\}^{n}$.

Доказателъство. Докажем достаточность. Пусть $\mathbf{F D E}^{\mathbf{x}}+$ - собственное дедуктивное расширение $\mathbf{F} \mathbf{D E}^{\mathbf{x}}$. Тогда найдутся такие множества формул $X$ и $Y$, что $h^{*}(X) \subseteq\{\mathbf{t}, \mathbf{b}\}$ и $h^{*}(Y) \subseteq\{\mathbf{n}, \mathbf{f}\}$ для некоторой оценки $h^{*}$ в $\mathcal{M}_{\mathbf{F D E}^{\mathbf{x}}}$, однако $X \vdash Y$ в $\mathbf{F D E}^{\mathbf{x}+}$. Пусть $\epsilon-$ подстановка, причем для каждой формулы $\gamma \in X$ и каждой формулы $\alpha \in Y$ верно, что $p_{0} \vdash \varepsilon(\gamma), q_{0}, \neg q_{0}$ и $\varepsilon(\alpha) \vdash q_{0}, \neg q_{0}$ в $\mathbf{F D E}^{\mathbf{x}}$. Поскольку $\mathbf{F D E}^{\mathbf{x}+}-$ собственное дедуктивное расширение $\mathbf{F D E}^{\mathbf{x}}, p_{0} \vdash \varepsilon(\gamma), q_{0}, \neg q_{0}$ для всех $\gamma \in X$ и $\varepsilon(\alpha) \vdash q_{0}, \neg q_{0}$ для всех $\alpha \in Y$ также в $\mathbf{F D E}^{\mathbf{x}}$. Кроме того, в силу структурности $\epsilon(X) \vdash \epsilon(Y)$ в $\mathbf{F D E}^{\mathbf{x}+}$. (1) Из $p_{0} \vdash \varepsilon(\gamma), q_{0}, \neg q_{0}$ получаем $p_{0}, Z \vdash(\epsilon(X) \cup \epsilon(Y)) \backslash Z, q_{0}, \neg q_{0}$ для любого такого $Z \subseteq \epsilon(X) \cup \epsilon(Y)$, что $\epsilon(\gamma) \in(\epsilon(X) \cup \epsilon(Y)) \backslash Z$ для некоторой формулы $\gamma \in X$ (монотонность). (2) Из $\varepsilon(\alpha) \vdash q_{0}, \neg q_{0}$ получаем $p_{0}, Z \vdash(\epsilon(X) \cup \epsilon(Y)) \backslash Z, q_{0}, \neg q_{0}$ для любого такого $Z \subseteq \epsilon(X) \cup \epsilon(Y)$, что $\epsilon(\alpha) \in Z$ для некоторой формулы $\alpha \in Y$ (монотонность). (3) Из $\epsilon(X) \vdash \epsilon(Y)$ получаем $p_{0}, Z \vdash(\epsilon(X) \cup \epsilon(Y)) \backslash Z, q_{0}, \neg q_{0}$ для $Z=\epsilon(X)$, то есть единственного такого $Z \subseteq \epsilon(X) \cup \epsilon(Y)$, что $\epsilon(\gamma) \notin(\epsilon(X) \cup \epsilon(Y)) \backslash Z$ для всех $\gamma \in X$ и $\epsilon(\alpha) \notin Z$ для всех $\alpha \in Y$ (монотонность). Из (1)-(3) получаем, что для всех $Z \subseteq \epsilon(X) \cup \epsilon(Y)$ имеет место $p_{0}, Z \vdash(\epsilon(X) \cup \epsilon(Y)) \backslash Z, q_{0}, \neg q_{0}$. Таким образом, $p_{0} \vdash q_{0}, \neg q_{0}$ в $\mathbf{F D E}^{\mathbf{x}+}$ (сечение). В силу последнего логика $\mathbf{F D E}^{\mathbf{x}+}$ не является параполной. Теперь покажем, что нужная подстановка $\epsilon$ всегда существует.

Случай 1 . В $\mathcal{M}_{\mathbf{F D E x}}$ содержится такая элементарная операция $g^{\prime}$, существенно зависящая от $n$ переменных, что $g^{\prime}(\mathbf{n}, \ldots, \mathbf{n}) \in\{\mathbf{t}, \mathbf{b}, \mathbf{f}\}$. Тогда в $\mathcal{M}_{\mathbf{F D E}}{ }^{\mathbf{0}}$ определима такая унарная операция $g$, что $g(\mathbf{n}) \in\{\mathbf{t}, \mathbf{b}, \mathbf{f}\}$. Всегда можно получить $g$ из $g^{\prime}$ отождествлением переменных. Это означает, что в $\mathcal{M}_{\mathbf{F D E}}{ }^{\mathbf{x}}$ можно также определить такую операцию $g^{*}$, что $g^{*}(\mathbf{n})=\mathbf{b}$. Покажем это. Если $g(\mathbf{n})=\mathbf{b}$, утверждение тривиально. Допустим, что $g(\mathbf{n})=\mathbf{t}$. По условию $\mathcal{M}_{\mathbf{F D E x}}$ содержит такую операцию $f$, что $f\left(a_{1}, \ldots, a_{n}\right)=\mathbf{b}$ на некотором наборе $\left(a_{1}, \ldots, a_{n}\right) \in\{\mathbf{t}, \mathbf{n}, \mathbf{f}\}^{n}$. Тогда $g^{*}=f\left(\varphi\left(x_{1}\right), \ldots, \varphi\left(x_{n}\right)\right)$, где для всех $i \in\{1, \ldots, n\}$ 


$$
\varphi\left(x_{i}\right)=\left\{\begin{array}{l}
g\left(x_{0}\right), \text { если } a_{i}=\mathbf{t}, \\
x_{0}, \text { если } a_{i}=\mathbf{n}, \\
\neg g\left(x_{0}\right), \text { если } a_{i}=\mathbf{f} .
\end{array}\right.
$$

Рассуждение для $g(\mathbf{n})=\mathbf{f}$ аналогично, достаточно поменять местами $g\left(x_{0}\right)$ и $\neg g\left(x_{0}\right)$ в определении $\varphi$. Пусть формула $\theta(p)$ выражает функцию $g^{*}\left(x_{0}\right)$, то есть $h(\theta(p))=g^{*}(h(p))$ для любой оценки $h$ в $\mathcal{M}_{\mathbf{F D E x}}$.

Обозначим как $\operatorname{Var}(X, Y)$ множество $\left\{q_{1}, \ldots, q_{k}\right\}$ всех переменных, входящих в формулы, принадлежащие $X$ или $Y$. Рассмотрим такую подстановку $\varepsilon$, что для каждой переменной $q \in \operatorname{Var}(X, Y)$ выполняется приведенное ниже условие:

$$
\varepsilon(q)=\left\{\begin{array}{l}
q_{0} \vee \theta\left(q_{0}\right), \text { если } h^{*}(q)=\mathbf{t}, \\
\theta\left(q_{0}\right), \text { если } h^{*}(q)=\mathbf{b}, \\
q_{0}, \text { если } h^{*}(q)=\mathbf{n}, \\
q_{0} \wedge \theta\left(q_{0}\right), \text { если } h^{*}(q)=\mathbf{f} .
\end{array}\right.
$$

Для каждой оценки $h$ в $\mathcal{M}_{\mathbf{F D E}}$ имеет место следующее: если $h\left(q_{0}\right)=\mathbf{n}$, то $h(\varepsilon(\gamma))=h^{*}(\gamma) \in\{\mathbf{t}, \mathbf{b}\}$ для всех $\gamma \in X$ и $h(\varepsilon(\alpha))=h^{*}(\alpha) \in\{\mathbf{n}, \mathbf{f}\}$ для всех $\alpha \in Y$. Обоснование этого утверждения строится по аналогии со Случаем 1 в доказательстве Теоремы 1. Это означает, что $\varepsilon(\alpha) \vdash q_{0}, \neg q_{0}$ и $p_{0} \vdash \varepsilon(\gamma), q_{0}, \neg q_{0}$ в $\mathbf{F D E}^{\mathbf{x}}$ для всех $\gamma \in X$ и всех $\alpha \in Y$.

Случай 2. Для каждой операции $g$ в $\mathcal{M}_{\mathbf{F D E}} \mathbf{x}^{\mathbf{x}}$ верно, что $g(\mathbf{n}, \ldots, \mathbf{n})=\mathbf{n}$. Поскольку $\mathcal{M}_{\mathbf{F D E}}$ содержит такую операцию $f$, что $f\left(a_{1}, \ldots, a_{n}\right)=\mathbf{b}$ на некотором наборе $\left(a_{1}, \ldots, a_{n}\right) \in\{\mathbf{t}, \mathbf{n}, \mathbf{f}\}^{n}$, найдется такая формула $\beta$, не содержащая переменных из $\operatorname{Var}(X, Y)$, что для некоторой оценки $h^{\mathbf{b}}$ в $\mathcal{M}_{\text {FDEx }}$ выполняется следующее условие: $h^{\mathbf{b}}(\beta)=\mathbf{b}$ и $h^{\mathbf{b}}(r) \in\{\mathbf{t}, \mathbf{n}, \mathbf{f}\}$ для каждой $r \in \operatorname{Var}(\beta)$, где $\operatorname{Var}(\beta)$ есть множество $\left\{r_{1}, \ldots, r_{m}\right\}$ всех переменных, входящих в $\beta$.

Рассмотрим такую подстановку $\varepsilon$, что для каждой переменной $r \in \operatorname{Var}(\beta)$ и каждой переменной $q \in \operatorname{Var}(X, Y)$ выполняются приведенные ниже условия:

$$
\begin{aligned}
& \varepsilon(r)=\left\{\begin{array}{l}
\left(p_{0} \vee q_{0}\right) \vee\left(\neg p_{0} \vee \neg q_{0}\right), \text { если } h^{\mathbf{b}}(r)=\mathbf{t}, \\
q_{0}, \text { если } h^{\mathbf{b}}(r)=\mathbf{n}, \\
\left(p_{0} \wedge q_{0}\right) \wedge\left(\neg p_{0} \wedge \neg q_{0}\right), \text { если } h^{\mathbf{b}}(r)=\mathbf{f} .
\end{array}\right. \\
& \varepsilon(q)=\left\{\begin{array}{l}
\left(p_{0} \vee q_{0}\right) \vee\left(\neg p_{0} \vee \neg q_{0}\right), \text { если } h^{*}(q)=\mathbf{t}, \\
\varepsilon(\beta), \text { если } h^{*}(q)=\mathbf{b}, \\
q_{0}, \text { если } h^{*}(q)=\mathbf{n}, \\
\left(p_{0} \wedge q_{0}\right) \wedge\left(\neg p_{0} \wedge \neg q_{0}\right), \text { если } h^{*}(q)=\mathbf{f} .
\end{array}\right.
\end{aligned}
$$


Для каждой оценки $h$ в $\mathcal{M}_{\mathbf{F D E}^{\mathbf{x}}}$ имеет место следующее: если $h\left(q_{0}\right)=\mathbf{n}$ и $h\left(p_{0}\right) \in\{\mathbf{t}, \mathbf{b}, \mathbf{f}\}$, то $h(\varepsilon(\gamma))=h^{*}(\gamma) \in\{\mathbf{t}, \mathbf{b}\}$ для всех $\gamma \in X$ и $h(\varepsilon(\alpha))=h^{*}(\alpha) \in\{\mathbf{n}, \mathbf{f}\}$ для всех $\alpha \in Y$. Обоснование этого утверждения строится по аналогии со Случаем 2 в доказательстве Теоремы 1. Кроме того, поскольку для каждой операции $g$ в $\mathcal{M}_{\mathbf{F D E x}}$ верно, что $g(\mathbf{n}, \ldots, \mathbf{n})=\mathbf{n}$, если $h\left(p_{0}\right)=h\left(q_{0}\right)=\mathbf{n}$, то $h(\varepsilon(\gamma))=h(\varepsilon(\alpha))=\mathbf{n}$ для всех $\gamma \in X$ и всех $\alpha \in Y$. Таким образом, снова $\varepsilon(\alpha) \vdash q_{0}, \neg q_{0}$ и $p_{0} \vdash \varepsilon(\gamma), q_{0}, \neg q_{0}$ в $\mathbf{F D E}^{\mathbf{x}}$ для всех $\gamma \in X$ и всех $\alpha \in Y$. Достаточность доказана.

Теперь докажем необходимость. Допустим, что для каждой операции $f$ в $\mathcal{M}_{\mathbf{F D E}^{x}}$ верно, что $f\left(a_{1}, \ldots, a_{n}\right) \in\{\mathbf{t}, \mathbf{n}, \mathbf{f}\}$ для каждого набора $\left(a_{1}, \ldots, a_{n}\right) \in\{\mathbf{t}, \mathbf{n}, \mathbf{f}\}^{n}$. В этом случае $\mathcal{M}_{\mathbf{F D E}}$ содержит подматрицу вида $\mathcal{M}_{\mathbf{K}_{3}^{\mathrm{x}}}=\left\langle\{\mathbf{t}, \mathbf{n}, \mathbf{f}\}, \wedge, \vee, \neg, f_{1}, \ldots, f_{k},\{\mathbf{t}\}\right\rangle$. Имеют место следующие факты: если $\langle X, Y\rangle \in C n\left(\mathcal{M}_{\mathbf{F D E}^{\mathbf{x}}}\right)$, то $\langle X, Y\rangle \in C n\left(\mathcal{M}_{\mathbf{K}_{3}^{\mathbf{x}}}\right)$; $\langle\{p, \neg p\},\{q, \neg q\}\rangle \notin C n\left(\mathcal{M}_{\mathbf{F D E}^{\mathbf{x}}}\right), \quad$ но $\langle\{p, \neg p\},\{q, \neg q\}\rangle \in C n\left(\mathcal{M}_{\mathbf{K}_{3}^{\mathbf{x}}}\right)$; $\langle\{p\},\{q, \neg q\}\rangle \notin C n\left(\mathcal{M}_{\mathbf{K}_{3}^{\mathbf{x}}}\right)$. Следовательно, трехзначная логика $\mathbf{K}_{\mathbf{3}}^{\mathbf{x}}$, для которой матрица $\mathcal{M}_{\mathbf{K}_{3}^{\mathrm{x}}}$ является характеристической, - это параполное собственное дедуктивное расширение $\mathbf{F D E}^{\mathbf{x}}$. Таким образом, $\mathbf{F D E}^{\mathbf{x}}$ не максимально параполна.

Полученные результаты позволяют ответить на вопрос, какие замкнутые классы функций могут быть порождены четырехзначными языковыми расширениями FDE, которые одновременно максимально паранепротиворечивы и максимально параполны. Этот ответ будет дан в следующем разделе.

\section{2. Замкнутые классы функций, порожденные максимально паранепротиворечивыми и параполными четырехзначными языковыми расширениями FDE}

Основным результатом раздела является Теорема 4. В ней мы приводим полный список замкнутых классов функций, которые могут быть порождены наборами элементарных операций матриц, характеризующих такие четырехзначные языковые расширения FDE, что в них совмещаются свойства максимальной паранепротиворечивости и максимальной параполноты. Кроме того, в Теореме 3 предложены критерии функциональной полноты и предполноты для произвольного четырехзначного расширения FDE. Содержание раздела имеет следующий вид. Сперва мы вводим ряд понятий, таких как сохранение функцией предиката, замкнутые классы функций и предикатов, понятие существенного предиката. Далее уделяется внимание тому факту, что замкнутый класс функций можно трактовать 
как класс всех функций, сохраняющих определенное множество существенных предикатов. Для замкнутого класса, порождаемого элементарными операциями характеристической матрицы FDE, такое множество конечно, причем местность предикатов, входящих в него не превышает 2. Как следствие, это верно и для всех надклассов данного класса, то есть замкнутых классов функций, порождаемых элементарными операциями матриц, которые являются характеристическими для четырехзначных языковых расширений FDE. Мы описываем множество $R_{\text {FDE }}$ всех существенных предикатов местности не более 2 , которые сохраняют операции $\mathcal{M}_{\mathbf{F D E}}$. На основе этого описания мы устанавливаем, какие классы, предполные в классе всех функций на множестве $\{\mathbf{t}, \mathbf{b}, \mathbf{n}, \mathbf{f}\}$, могут быть порождены расширениями FDE, что позволяет сформулировать и доказать Теорему 3 . После этого доказываются Леммы 175, на которые опирается доказательство Теоремы 4, и сама основная теорема. Раздел завершает диаграмма, на которой замкнутые классы, представленные в основной теореме, упорядочены по отношению включения.

Если дано множество $A$, назовем отображение $A^{n} \longmapsto\{0,1\} n$-местным предикатом на $A$. Для удобства будем записывать предикаты в форме матриц вида

$$
\varrho=\left(\begin{array}{cccc}
a_{1,1} & a_{2,1} & \ldots & a_{m, 1} \\
a_{1,2} & a_{2,2} & \ldots & a_{m, 2} \\
\vdots & \vdots & \ddots & \vdots \\
a_{1, n} & a_{2, n} & \ldots & a_{m, n}
\end{array}\right)
$$

где $\varrho\left(a_{i, 1}, \ldots, a_{i, n}\right)=1$ для всех $i \in\{1, \ldots, m\}$ и $\varrho$ принимает значение 0 на всех прочих наборах. В случаях, когда это не повлечет нежелательных последствий, будем рассматривать предикаты как отношения и писать $\left(a_{1}, \ldots, a_{n}\right) \in \varrho$ вместо $\varrho\left(a_{1}, \ldots, a_{n}\right)=1$.

Говорим, что $k$-местная функция $f$ сохраняет предикат $\varrho$, если

$$
f\left(\begin{array}{cccc}
b_{1,1} & b_{2,1} & \ldots & b_{k, 1} \\
b_{1,2} & b_{2,2} & \ldots & b_{k, 2} \\
\vdots & \vdots & \ddots & \vdots \\
b_{1, n} & b_{2, n} & \ldots & b_{k, n}
\end{array}\right)=\left(\begin{array}{c}
f\left(b_{1,1}, b_{2,1}, \ldots, b_{k, 1}\right) \\
f\left(b_{1,2}, b_{2,2}, \ldots, b_{k, 2}\right) \\
\vdots \\
f\left(b_{1, n}, b_{2, n}, \ldots, b_{k, n}\right)
\end{array}\right) \in \varrho
$$

коль скоро $\varrho\left(b_{i, 1}, \ldots, b_{i, n}\right)=1$ для каждого $i \in\{1, \ldots, k\}$. Если даны множество функций $F$ и множество предикатов $R$, говорим, что $F$ coхраняет $R$, если всякая функция из $F$ сохраняет всякий предикат из $R$.

Обозначаем как $\operatorname{Inv}(F)$ множество всех предикатов, которые сохраняет каждая функция из $F$, и как $\operatorname{Pol}(R)$ - множество всех функций, которые сохраняют каждый предикат из $R$. 
Введем понятия замыкания класса функций и замыкания класса предикатов следующим образом: $[F]=\operatorname{Pol}(\operatorname{Inv}(F)) ;[R]=\operatorname{Inv}(\operatorname{Pol}(R))$. Для краткости вместо прямых определений, в которых не задействованы $\mathrm{Pol}$ и Inv, мы используем результаты теории Галуа для замкнутых классов (cм. [Lau, 2006, p. 132, 135]). Стандартные определения этих понятий можно найти в (Lau, 2006, P. II, §§1.2, 2.3-2.4]).

Называем предикат @ существенным, если не существует таких предикатов $\varrho_{1}, \ldots, \varrho_{n}$, что $\operatorname{ar}\left(\varrho_{i}\right)<\operatorname{ar}(\varrho)$ для любого $i \in\{1, \ldots, n\}$, и при этом $[\{\varrho\}]=\left[\left\{\varrho_{1}, \ldots, \varrho_{n}\right\}\right] \mid$ Жук, 2011].

Пусть функция $f$ выполняет следующее условие: $f(x, x, y)=$ $f(x, y, x)=f(y, x, x)=x$. В этом случае называем $f$ функиией голосования. Как указывает Д.Н. Жук ЖКук, 2018] со ссылкой на работу Baker, Pixley, 1975], для любого замкнутого класса $F$, содержащего функцию голосования, выполняется тождество $F=\operatorname{Pol}(R)$, где $1 \leq \operatorname{ar}(\varrho) \leq 2$ для каждого $\varrho \in R$. Действительно, соответствующее утверждение (Лемма 3 ) в [Жук, 2018] является частным случаем результата, изложенного в Baker, Pixley, 1975, § 5.1]. В силу Леммы 2 из Жук, 2011 также верно, что любой замкнутый класс, содержащий функцию голосования, может быть задан множеством только существенных предикатов арности 1 или 2.

Будем обозначать замкнутый класс функций, порожденный элементарными операциями матрицы $\mathcal{M}_{\mathbf{F D E}}$, как $[F D E]^{8}$ и замкнутый класс функций, порожденный элементарными операциями матрицы $\mathcal{M}_{\mathbf{F D E x}},-$ как $\left[F D E^{x}\right]$. Нетрудно убедиться, что $[F D E]$ и, как следствие, $\left[F D E^{x}\right]$ содержат функцию голосования:

$$
f(x, y, z)=(x \vee y) \wedge(x \vee z) \wedge(y \vee z) .
$$

Поэтому $[F D E]=\operatorname{Pol}\left(R_{F D E}\right)$, где $R_{F D E}-$ множество существенных предикатов местности 1 или 2.

Ниже мы приводим полный список существенных предикатов местности 1 и 2, которые сохраняет каждая функция $[F D E]$. Он был получен перебором с помощью компьютерной программы.

$$
\begin{gathered}
\varrho_{1}^{1}=(\mathbf{n}), \varrho_{2}^{1}=(\mathbf{b}), \varrho_{3}^{1}=(\mathbf{t}, \mathbf{f}), \varrho_{4}^{1}=(\mathbf{t}, \mathbf{n}, \mathbf{f}), \varrho_{5}^{1}=(\mathbf{t}, \mathbf{b}, \mathbf{f}), \\
\varrho_{1}^{2}=\left(\begin{array}{llll}
\mathbf{t} & \mathbf{f} & \mathbf{b} & \mathbf{n} \\
\mathbf{t} & \mathbf{f} & \mathbf{n} & \mathbf{b}
\end{array}\right), \varrho_{2}^{2}=\left(\begin{array}{ccc}
\mathbf{t} & \mathbf{f} & \mathbf{n} \\
\mathbf{t} & \mathbf{f} & \mathbf{b}
\end{array}\right), \varrho_{3}^{2}=\left(\begin{array}{cccc}
\mathbf{t} & \mathbf{f} & \mathbf{n} & \mathbf{n} \\
\mathbf{t} & \mathbf{f} & \mathbf{t} & \mathbf{f}
\end{array}\right), \\
\partial \varrho_{3}^{2}=\left(\begin{array}{llll}
\mathbf{t} & \mathbf{f} & \mathbf{b} & \mathbf{b} \\
\mathbf{t} & \mathbf{f} & \mathbf{t} & \mathbf{f}
\end{array}\right), \varrho_{4}^{2}=\left(\begin{array}{lllll}
\mathbf{t} & \mathbf{f} & \mathbf{n} & \mathbf{n} & \mathbf{n} \\
\mathbf{t} & \mathbf{f} & \mathbf{n} & \mathbf{t} & \mathbf{f}
\end{array}\right), \partial \varrho_{4}^{2}=\left(\begin{array}{ccccc}
\mathbf{t} & \mathbf{f} & \mathbf{b} & \mathbf{b} & \mathbf{b} \\
\mathbf{t} & \mathbf{f} & \mathbf{b} & \mathbf{t} & \mathbf{f}
\end{array}\right),
\end{gathered}
$$

\footnotetext{
8Заметим, что $[F D E]=[\wedge, \neg]$, так как $x \vee y=\neg(\neg x \wedge \neg y)$.
} 


$$
\begin{aligned}
& \varrho_{5}^{2}=\left(\begin{array}{ccccc}
\mathbf{t} & \mathbf{f} & \mathbf{n} & \mathbf{n} & \mathbf{n} \\
\mathbf{t} & \mathbf{f} & \mathbf{t} & \mathbf{b} & \mathbf{f}
\end{array}\right), \partial \varrho_{5}^{2}=\left(\begin{array}{ccccc}
\mathbf{t} & \mathbf{f} & \mathbf{b} & \mathbf{b} & \mathbf{b} \\
\mathbf{t} & \mathbf{f} & \mathbf{t} & \mathbf{n} & \mathbf{f}
\end{array}\right) \\
& \varrho_{6}^{2}=\left(\begin{array}{cccccc}
\mathbf{t} & \mathbf{f} & \mathbf{n} & \mathbf{n} & \mathbf{n} & \mathbf{n} \\
\mathbf{t} & \mathbf{f} & \mathbf{n} & \mathbf{t} & \mathbf{b} & \mathbf{f}
\end{array}\right), \partial \varrho_{6}^{2}=\left(\begin{array}{cccccc}
\mathbf{t} & \mathbf{f} & \mathbf{b} & \mathbf{b} & \mathbf{b} & \mathbf{b} \\
\mathbf{t} & \mathbf{f} & \mathbf{b} & \mathbf{t} & \mathbf{n} & \mathbf{f}
\end{array}\right) \\
& \varrho_{7}^{2}=\left(\begin{array}{ccccccc}
\mathbf{t} & \mathbf{f} & \mathbf{n} & \mathbf{t} & \mathbf{n} & \mathbf{f} & \mathbf{n} \\
\mathbf{t} & \mathbf{f} & \mathbf{n} & \mathbf{n} & \mathbf{t} & \mathbf{n} & \mathbf{f}
\end{array}\right), \partial \varrho_{7}^{2}=\left(\begin{array}{ccccccc}
\mathbf{t} & \mathbf{f} & \mathbf{b} & \mathbf{t} & \mathbf{b} & \mathbf{f} & \mathbf{b} \\
\mathbf{t} & \mathbf{f} & \mathbf{b} & \mathbf{b} & \mathbf{t} & \mathbf{b} & \mathbf{f}
\end{array}\right), \\
& \varrho_{8}^{2}=\left(\begin{array}{ccccccc}
\mathbf{t} & \mathbf{f} & \mathbf{t} & \mathbf{f} & \mathbf{n} & \mathbf{n} & \mathbf{n} \\
\mathbf{t} & \mathbf{f} & \mathbf{b} & \mathbf{b} & \mathbf{t} & \mathbf{b} & \mathbf{f}
\end{array}\right), \varrho_{9}^{2}=\left(\begin{array}{cccccccc}
\mathbf{t} & \mathbf{f} & \mathbf{n} & \mathbf{t} & \mathbf{f} & \mathbf{n} & \mathbf{n} & \mathbf{n} \\
\mathbf{t} & \mathbf{f} & \mathbf{n} & \mathbf{n} & \mathbf{n} & \mathbf{t} & \mathbf{b} & \mathbf{f}
\end{array}\right), \\
& \partial \varrho_{9}^{2}=\left(\begin{array}{cccccccc}
\mathbf{t} & \mathbf{f} & \mathbf{b} & \mathbf{t} & \mathbf{f} & \mathbf{b} & \mathbf{b} & \mathbf{b} \\
\mathbf{t} & \mathbf{f} & \mathbf{b} & \mathbf{b} & \mathbf{b} & \mathbf{t} & \mathbf{n} & \mathbf{f}
\end{array}\right), \varrho_{10}^{2}=\left(\begin{array}{cccccccc}
\mathbf{t} & \mathbf{f} & \mathbf{n} & \mathbf{t} & \mathbf{f} & \mathbf{b} & \mathbf{b} & \mathbf{b} \\
\mathbf{t} & \mathbf{f} & \mathbf{n} & \mathbf{n} & \mathbf{n} & \mathbf{t} & \mathbf{n} & \mathbf{f}
\end{array}\right), \\
& \partial \varrho_{10}^{2}=\left(\begin{array}{cccccccc}
\mathbf{t} & \mathbf{f} & \mathbf{b} & \mathbf{t} & \mathbf{f} & \mathbf{n} & \mathbf{n} & \mathbf{n} \\
\mathbf{t} & \mathbf{f} & \mathbf{b} & \mathbf{b} & \mathbf{b} & \mathbf{t} & \mathbf{b} & \mathbf{f}
\end{array}\right), \varrho_{11}^{2}=\left(\begin{array}{ccccccccc}
\mathbf{t} & \mathbf{f} & \mathbf{n} & \mathbf{t} & \mathbf{n} & \mathbf{n} & \mathbf{n} & \mathbf{b} & \mathbf{f} \\
\mathbf{t} & \mathbf{f} & \mathbf{n} & \mathbf{n} & \mathbf{t} & \mathbf{b} & \mathbf{f} & \mathbf{n} & \mathbf{n}
\end{array}\right), \\
& \partial \rho_{11}^{2}=\left(\begin{array}{ccccccccc}
\mathbf{t} & \mathbf{f} & \mathbf{b} & \mathbf{t} & \mathbf{b} & \mathbf{b} & \mathbf{b} & \mathbf{n} & \mathbf{f} \\
\mathbf{t} & \mathbf{f} & \mathbf{b} & \mathbf{b} & \mathbf{t} & \mathbf{n} & \mathbf{f} & \mathbf{b} & \mathbf{b}
\end{array}\right), \\
& \rho_{12}^{2}=\left(\begin{array}{ccccccccc}
\mathbf{t} & \mathbf{f} & \mathbf{b} & \mathbf{n} & \mathbf{t} & \mathbf{f} & \mathbf{n} & \mathbf{n} & \mathbf{n} \\
\mathbf{t} & \mathbf{f} & \mathbf{b} & \mathbf{n} & \mathbf{b} & \mathbf{b} & \mathbf{t} & \mathbf{b} & \mathbf{f}
\end{array}\right)
\end{aligned}
$$

Таким образом, $R_{F D E}=\left\{\varrho_{1}^{1}, \ldots, \varrho_{5}^{1}, \varrho_{1}^{2}, \ldots, \varrho_{12}^{2}, \partial \varrho_{4}^{2}, \ldots, \partial \varrho_{11}^{2}\right\}$.

Здесь мы сделаем небольшое отступление, касающееся проблемы функциональной полноты в матрицах четырехзначных языковых расширений FDE. Пусть даны множества функций $F$ и $G$. Говорим, что множество $F$ функиионально полно в $G$, если $[F]=G$. Называем множество $F$ предполным в $G$, если $F$ не полно в $G$, однако $[F \cup\{g\}]=G$ для всех $g \in G \backslash F$. Обозначим как $P_{\{\mathbf{t}, \mathbf{b}, \mathbf{n}, \mathbf{f}\}}$ класс всех функций на множестве $\{\mathbf{t}, \mathbf{b}, \mathbf{n}, \mathbf{f}\}$. Вопрос об условиях полноты системы элементарных операций матрицы, задающей четырехзначное языковое расширение FDE, неоднократно поднимался в литературе. Доказательства функциональной полноты или предполноты для отдельных примеров даются в Arieli, Avron, 1998; Avron, 1999, Arieli, Avron, 2017; Přenosil, 2021. Более общие достаточные условия функциональной полноты, данные в терминах выразимости тех или иных функций в матрицах, можно найти в Pynko, 1999; Omori, Sano, 2015]. Полученный нами результат позволяет внести новый вклад в исследование проблемы полноты раширений FDE, сформулировав необходимые и достаточные условия в терминах сохранения операциями матриц классов предикатов.

Теорема 3. Пусть $\mathcal{M}_{\mathbf{F D E}^{\mathbf{x}}}=\left\langle\{\mathbf{t}, \mathbf{b}, \mathbf{n}, \mathbf{f}\}, \wedge, \vee, \neg, f_{1}, \ldots, f_{k},\{\mathbf{t}, \mathbf{b}\}\right\rangle-x a-$ рактеристическая матрица языкового расширения FDE. 
(1) Система функииц $\left\{\wedge, \vee, \neg, f_{1}, \ldots, f_{k}\right\}$ функционально полна в $P_{\{\mathbf{t}, \mathbf{b}, \mathbf{n}, \mathbf{f}\}}$, е.т.е. для каждого $\varrho \in\left\{\varrho_{1}^{1}, \ldots, \varrho_{5}^{1}, \varrho_{12}^{2}\right\}$ найдется такая функиия $f_{i}(1 \leq i \leq k)$, ито $f_{i} \notin \operatorname{Pol}(\varrho)^{9}$.

(2) Система функиий $\left\{\wedge, \vee, \neg, f_{1}, \ldots, f_{k}\right\}$ предполна в $P_{\{\mathbf{t}, \mathbf{b}, \mathbf{n}, \mathbf{f}\}}$, e.m.e. существует в точности один такой предикат $\varrho \in\left\{\varrho_{1}^{1}, \ldots, \varrho_{5}^{1}, \varrho_{12}^{2}\right\}$, что для каждой функиии $f_{i}(1 \leq i \leq k)$ выполняется $f_{i} \in \operatorname{Pol}(\varrho)$.

Доказателъство. Известно, что система функций $k$-значной логики является функционально полной в классе всех функций на $k$-элементном множестве, е.т.е. она не содержится полностью ни в одном из замкнутых классов функций, предполных в этом классе [Lau, 2006, p. 91]. Предикаты, задающие каждый замкнутый класс, предполный в классе всех функций на четырехэлементном множестве, даны в Нагорный, 2013, с. 105107]. Пересечение множества этих предикатов со множеством $R_{F D E}$ дает $\left\{\varrho_{1}^{1}, \ldots, \varrho_{5}^{1}, \varrho_{12}^{2}\right\}$, что демонстрирует истинность утверждения (1). Далее, истинность утверждения (2) вытекает из того факта, что ни один предполный в $P_{\{\mathbf{t}, \mathbf{b}, \mathbf{n}, \mathbf{f}\}}$ класс не может целиком содержаться ни в каком другом замкнутом классе, кроме $P_{\{\mathbf{t}, \mathbf{b}, \mathbf{n}, \mathbf{f}\}}$.

Возвращаемся к основному предмету работы - вопросу о том, какие замкнутые классы могут быть порождены языковыми расширениями FDE, которые одновременно максимально паранепротиворечивы и максимально параполны. В силу теории Галуа для замкнутых классов, из $[F D E] \subseteq\left[F D E^{x}\right]$ вытекает $\operatorname{Inv}\left(\left[F D E^{x}\right]\right) \subseteq \operatorname{Inv}([F D E])$ Lau, 2006, Th. 2.9.3]. Так как $[F D E]=\operatorname{Pol}\left(R_{F D E}\right)$, это влечет $\left[F D E^{x}\right]=\operatorname{Pol}\left(R_{F D E^{x}}\right) \Longrightarrow R_{F D E^{x}} \subseteq R_{F D E}$, где $R_{F D E^{x}}-$ множество всех существенных предикатов местности 1 и 2 , которые сохраняет $\left[F D E^{x}\right]$. Теперь остается определить, какие именно подмножества $R_{F D E}$ описывают замкнутые классы функций, соответствующие интересующим нас расширениям FDE.

Лемма 1. FDE $^{\mathbf{x}}$ максимально паранепротиворечива, е.т.е. $\mathcal{M}_{\mathbf{F D E}^{\mathbf{x}}}$ содержит операчию, не сохраняющую предикат $\varrho_{5}^{1}$.

Доказательство. Вытекает из Теоремы 1 и $\varrho_{5}^{1}=\left(\begin{array}{lll}\mathbf{t} & \mathbf{b} & \mathbf{f}\end{array}\right)$.

Лемма 2. $\mathbf{F D E}^{\mathbf{x}}$ максимально параполна, е.т.е. $\mathcal{M}_{\mathbf{F D E}^{\mathbf{x}}}$ содержит операчию, не сохраняющую предикат $\varrho_{4}^{1}$.

Доказательство. Вытекает из Теоремы 2 и $\varrho_{4}^{1}=\left(\begin{array}{lll}\mathbf{t} & \mathbf{n} & \mathbf{f}\end{array}\right)$.

\footnotetext{
${ }^{9}$ Условимся писать $\operatorname{Pol}(\varrho)$ вместо $\operatorname{Pol}(\{\varrho\})$, когда речь идет об одноэлементных множествах предикатов
} 
Лемма 3. $f \in \operatorname{Pol}\left(\varrho_{i}^{2}\right) \Longrightarrow f \in \operatorname{Pol}\left(\varrho_{4}^{1}\right)$ для $2 \leq i \leq 11$; $f \in \operatorname{Pol}\left(\partial \varrho_{i}^{2}\right) \Longrightarrow f \in \operatorname{Pol}\left(\varrho_{5}^{1}\right) \partial \Omega \Omega 3 \leq i \leq 11$.

Доказателъство. Пусть одна из строк матрицы предиката $\varrho$ содержит только три значения. Например, t, $\mathbf{f}$ и $\mathbf{b}$. Допустим, существует такая функция $f$, что она сохраняет предикат $\varrho$ и не сохраняет предикат $\varrho_{5}^{1}$. В этом случае $f\left(a_{1}, \ldots, a_{n}\right)=\mathbf{n}$ на некотором наборе $\left(a_{1}, \ldots, a_{n}\right) \in\{\mathbf{t}, \mathbf{b}, \mathbf{f}\}^{n}$. Чтобы при этом имело место $f \in \operatorname{Pol}(\varrho)$, для каждой строки матрицы $\varrho$ должно выполняться следующее условие: если строка содержит одновременно $\mathbf{t}, \mathbf{f}$ и $\mathbf{b}$, то она содержит также $\mathbf{n}$. Но это противоречит условию. Аналогичным образом, если хотя бы одна строка матрицы предиката $\varrho$ состоит из значений $\mathbf{t}, \mathbf{f}$ и $\mathbf{n}$, это означает, что $f \in \operatorname{Pol}(\varrho) \Longrightarrow f \in \operatorname{Pol}\left(\varrho_{4}^{1}\right)$. Лемма доказана для всех предикатов из условия, кроме $\varrho_{11}^{2}$ и $\partial \varrho_{11}^{2}$.

Теперь пусть среди столбцов матрицы предиката $\varrho$ в точности три состоят из одного и того же значения. Например, это столбцы $\left(\begin{array}{l}\mathbf{t} \\ \mathbf{t}\end{array}\right),\left(\begin{array}{l}\mathbf{f} \\ \mathbf{f}\end{array}\right)$, $\left(\begin{array}{l}\mathbf{b} \\ \mathbf{b}\end{array}\right)$. Допустим, существует такая функция $f$, что она сохраняет предикат $\varrho$ и не сохраняет предикат $\varrho_{5}^{1}$. В этом случае $f\left(a_{1}, \ldots, a_{n}\right)=\mathbf{n}$ на некотором наборе $\left(a_{1}, \ldots, a_{n}\right) \in\{\mathbf{t}, \mathbf{b}, \mathbf{f}\}^{n}$. Чтобы при этом имело место $f \in \operatorname{Pol}(\varrho)$, для матрицы $\varrho$ должно выполняться следующее условие: если матрица содержит одновременно $\left(\begin{array}{l}\mathbf{t} \\ \mathbf{t}\end{array}\right),\left(\begin{array}{l}\mathbf{f} \\ \mathbf{f}\end{array}\right),\left(\begin{array}{l}\mathbf{b} \\ \mathbf{b}\end{array}\right)$, то она содержит также $\left(\begin{array}{l}\mathbf{n} \\ \mathbf{n}\end{array}\right)$. Но это противоречит условию. Данное построение доказывает лемму для $\partial \varrho_{11}^{2}$. Доказательство для $\varrho_{11}^{2}$ аналогично, достаточно в рассуждении поменять местами значения $\mathbf{b}$ и $\mathbf{n}$, а также заменить $\varrho_{5}^{1}$ на $\varrho_{4}^{1}$.

Лемма 4. $f \in \operatorname{Pol}\left(\varrho_{3}^{1}\right) \cap \operatorname{Pol}\left(\varrho_{12}^{2}\right) \Longrightarrow f \in \operatorname{Pol}\left(\varrho_{4}^{1}\right) \cap \operatorname{Pol}\left(\varrho_{5}^{1}\right)$.

Доказательство. Класс $\operatorname{Pol}\left(\varrho_{3}^{1}\right)$ - это класс всех алгебраических функций на $\{\mathbf{t}, \mathbf{f}\}$, принадлежащих $P_{\{\mathbf{t}, \mathbf{b}, \mathbf{n}, \mathbf{f}\}}$. Класс $\operatorname{Pol}\left(\varrho_{12}^{2}\right)-$ это класс всех функций, монотонных относительно порядка $\leq_{k}$, где $\mathbf{n} \leq_{k} \mathbf{t} \leq_{k} \mathbf{b}$, $\mathbf{n} \leq_{k} \mathbf{f} \leq_{k}$ b. Порождающая система для $\operatorname{Pol}\left(\varrho_{3}^{1}\right) \cap \operatorname{Pol}\left(\varrho_{12}^{2}\right)$ приводится в Přenosil, 2021, Th. 18]. Все функции этой системы сохраняют как $\varrho_{4}^{1}$, так и $\varrho_{5}^{1}$.

Лемма 5. $f \in \operatorname{Pol}\left(\varrho_{1}^{2}\right) \Longrightarrow f \in \operatorname{Pol}\left(\varrho_{3}^{1}\right) ; f \in \operatorname{Pol}\left(\varrho_{1}^{2}\right) \Longrightarrow\left(f \in \operatorname{Pol}\left(\varrho_{1}^{1}\right) \Longleftrightarrow\right.$ $\left.f \in \operatorname{Pol}\left(\varrho_{2}^{1}\right)\right)$. 
Доказательство. Класс $\operatorname{Pol}\left(\varrho_{1}^{2}\right)$ - это класс всех функций, самодвойственных относительно $\partial$, где $\partial(\mathbf{t})=\mathbf{t}, \partial(\mathbf{b})=\mathbf{n}, \partial(\mathbf{n})=\mathbf{b}, \partial(\mathbf{f})=\mathbf{f}$. Порождающая система для $\operatorname{Pol}\left(\varrho_{1}^{2}\right)$ приводится в [Přenosil, 2021, Th. 5]. Все функции этой системы сохраняют $\varrho_{3}^{1}$, что демонстрирует истинность первого утверждения. Докажем второе утверждение. Пусть $f \in \operatorname{Pol}\left(\varrho_{1}^{2}\right)$, $f \in \operatorname{Pol}\left(\varrho_{1}^{1}\right)$ и $f \notin \operatorname{Pol}\left(\varrho_{2}^{1}\right)$, то есть $f(\mathbf{b}, \ldots, \mathbf{b}) \in\{\mathbf{t}, \mathbf{n}, \mathbf{f}\}$. Однако, как это следует из определения $\varrho_{1}^{2}$, в этом случае либо $f(\mathbf{n}, \ldots, \mathbf{n}) \in\{\mathbf{t}, \mathbf{b}, \mathbf{f}\}$, то есть $f \notin \operatorname{Pol}\left(\varrho_{1}^{1}\right)$, либо $f \notin \operatorname{Pol}\left(\varrho_{1}^{2}\right)$. В обоих случаях получаем противоречие. Рассуждение для случая $f \in \operatorname{Pol}\left(\varrho_{1}^{2}\right), f \in \operatorname{Pol}\left(\varrho_{2}^{1}\right), f \notin \operatorname{Pol}\left(\varrho_{1}^{1}\right)$ аналогично, достаточно поменять местами $\mathbf{b}$ и $\mathbf{n}$.

Теорема 4. Существует в точности 14 замкнутых классов функиий, которые могут быть порождены наборами элементарных операчий четырехзначных матрич, характеризующих максимально паранепротиворечивые и максимально параполные языковые расширения FDE. Это классы функи,ии, сохраняющие одно из следуюших множеств предикатов: $R_{1}=\{\varnothing\} ; R_{2}=\left\{\varrho_{12}^{2}\right\} ; R_{3}=\left\{\varrho_{1}^{1}, \varrho_{12}^{2}\right\} ; R_{4}=\left\{\varrho_{2}^{1}, \varrho_{12}^{2}\right\} ; R_{5}=\left\{\varrho_{1}^{1}, \varrho_{2}^{1}, \varrho_{12}^{2}\right\} ;$ $R_{6}=\left\{\varrho_{1}^{1}\right\} ; R_{7}=\left\{\varrho_{3}^{1}\right\} ; R_{8}=\left\{\varrho_{3}^{1}, \varrho_{1}^{2}\right\} ; R_{9}=\left\{\varrho_{2}^{1}\right\} ; R_{10}=\left\{\varrho_{1}^{1}, \varrho_{2}^{1}\right\} ;$ $R_{11}=\left\{\varrho_{1}^{1}, \varrho_{2}^{1}, \varrho_{3}^{1}, \varrho_{1}^{2}\right\} ; R_{12}=\left\{\varrho_{1}^{1}, \varrho_{3}^{1}\right\} ; R_{13}=\left\{\varrho_{2}^{1}, \varrho_{3}^{1}\right\} ; R_{14}=\left\{\varrho_{1}^{1}, \varrho_{2}^{1}, \varrho_{3}^{1}\right\}$.

Доказательство. Пусть $\mathbf{F D E}^{\mathbf{x}}$ - максимально паранепротиворечивое и максимально параполное четырехзначное языковое расширение FDE и $\left[F D E^{x}\right]=\operatorname{Pol}\left(R_{F D E^{x}}\right)$. В силу Лемм $13 R_{F D E^{x}} \subseteq\left\{\varrho_{1}^{1}, \varrho_{2}^{1}, \varrho_{3}^{1}, \varrho_{1}^{2}, \varrho_{12}^{2}\right\}$. В силу Леммы $4 \varrho_{3}^{1} \notin R_{F D E^{x}}$ или $\varrho_{12}^{2} \notin R_{F D E^{x}}$.

Пусть $\varrho_{12}^{2} \notin R_{F D E^{x}}$. Тогда $R_{F D E^{x}} \subseteq\left\{\varrho_{1}^{1}, \varrho_{2}^{1}, \varrho_{3}^{1}, \varrho_{1}^{2}\right\}$. Из Леммы 5 вытекает $\varrho_{1}^{2} \in R_{F D E^{x}} \Longrightarrow \varrho_{3}^{1} \in R_{F D E^{x}}$, а также $\left\{\varrho_{1}^{1}, \varrho_{1}^{2}\right\} \subseteq R_{F D E^{x}} \Longrightarrow \varrho_{2}^{1} \in R_{F D E^{x}}$ и $\left\{\varrho_{2}^{1}, \varrho_{1}^{2}\right\} \subseteq R_{F D E^{x}} \Longrightarrow \varrho_{1}^{1} \in R_{F D E^{x}}$. Поэтому $R_{F D E^{x}} \in\left\{R_{1}, R_{6}, R_{7}, R_{8}, R_{9}, R_{10}, R_{11}, R_{12}, R_{13}, R_{14}\right\}$. Теперь пусть $\varrho_{12}^{2} \in R_{F D E^{x}}$. Тогда $\varrho_{3}^{1} \notin R_{F D E^{x}}$. В силу Леммы $5 \varrho_{1}^{2} \notin R_{F D E^{x}}$. Следовательно, $R_{F D E^{x}} \in\left\{R_{2}, R_{3}, R_{4}, R_{5}\right\}$. Таким образом, мы показали, что $R_{F D E^{x}} \in\left\{R_{1}, \ldots, R_{14}\right\}$.

Покажем, что для каждого $R_{i} \in\left\{R_{1}, \ldots, R_{14}\right\}$ найдется такая логика $\mathbf{F D E}^{\mathbf{x}}$, что $\left[F D E^{x}\right]=\operatorname{Pol}\left(R_{i}\right)$. Примеры для $i \in\{1, \ldots, 7\}$ заимствуем из Avron, 1999 (см. Теоремы 3.10, 3.11, 3.15, 3.22-3.24). Пусть операции つ, $\otimes, \oplus$ отвечают таблицам, приведенным ниже.

\begin{tabular}{c|cccc}
$\supset$ & $\mathbf{t}$ & $\mathbf{b}$ & $\mathbf{n}$ & $\mathbf{f}$ \\
\hline $\mathbf{t}$ & $\mathbf{t}$ & $\mathbf{b}$ & $\mathbf{n}$ & $\mathbf{f}$ \\
$\mathbf{b}$ & $\mathbf{t}$ & $\mathbf{b}$ & $\mathbf{n}$ & $\mathbf{f}$ \\
$\mathbf{n}$ & $\mathbf{t}$ & $\mathbf{t}$ & $\mathbf{t}$ & $\mathbf{t}$ \\
$\mathbf{f}$ & $\mathbf{t}$ & $\mathbf{t}$ & $\mathbf{t}$ & $\mathbf{t}$
\end{tabular}

\begin{tabular}{c|cccc}
$\otimes$ & $\mathbf{t}$ & $\mathbf{b}$ & $\mathbf{n}$ & $\mathbf{f}$ \\
\hline $\mathbf{t}$ & $\mathbf{t}$ & $\mathbf{t}$ & $\mathbf{n}$ & $\mathbf{n}$ \\
$\mathbf{b}$ & $\mathbf{t}$ & $\mathbf{b}$ & $\mathbf{n}$ & $\mathbf{f}$ \\
$\mathbf{n}$ & $\mathbf{n}$ & $\mathbf{n}$ & $\mathbf{n}$ & $\mathbf{n}$ \\
$\mathbf{f}$ & $\mathbf{n}$ & $\mathbf{f}$ & $\mathbf{n}$ & $\mathbf{f}$
\end{tabular}

\begin{tabular}{c|cccc}
$\oplus$ & $\mathbf{t}$ & $\mathbf{b}$ & $\mathbf{n}$ & $\mathbf{f}$ \\
\hline $\mathbf{t}$ & $\mathbf{t}$ & $\mathbf{b}$ & $\mathbf{t}$ & $\mathbf{b}$ \\
$\mathbf{b}$ & $\mathbf{b}$ & $\mathbf{b}$ & $\mathbf{b}$ & $\mathbf{b}$ \\
$\mathbf{n}$ & $\mathbf{t}$ & $\mathbf{b}$ & $\mathbf{n}$ & $\mathbf{f}$ \\
$\mathbf{f}$ & $\mathbf{b}$ & $\mathbf{b}$ & $\mathbf{f}$ & $\mathbf{f}$
\end{tabular}


Тогда имеет место следующее:

1. $[\{\wedge, \neg, \supset, \mathbf{b}, \mathbf{n}\}]-$ это класс $P_{\{\mathbf{t}, \mathbf{b}, \mathbf{n}, \mathbf{f}\}}$ всех функций на множестве $\{\mathbf{t}, \mathbf{b}, \mathbf{n}, \mathbf{f}\}$, то есть $\operatorname{Pol}\left(R_{1}\right)$.

2. $[\{\wedge, \neg, \otimes, \oplus, \mathbf{b}, \mathbf{n}\}]-$ это подкласс $P_{\{\mathbf{t}, \mathbf{b}, \mathbf{n}, \mathbf{f}\}}$, состоящий из всех $\leq_{k}$-монотонных функций, то есть $\operatorname{Pol}\left(R_{2}\right)$.

3. $[\{\wedge, \neg, \otimes, \mathbf{b}\}]$ - это подкласс $P_{\{\mathbf{t}, \mathbf{b}, \mathbf{n}, \mathbf{f}\}}$, состоящий из всех $\leq_{k}$-монотонных функций, которые являются алгебраическими функциями на $\{\mathbf{b}\}$, то есть $\operatorname{Pol}\left(R_{3}\right)$.

4. $[\{\wedge, \neg, \oplus, \mathbf{n}\}]-$ это подкласс $P_{\{\mathbf{t}, \mathbf{b}, \mathbf{n}, \mathbf{f}\}}$, состоящий из всех $\leq_{k}$-монотонных функций, которые являются алгебраическими функциями на $\{\mathbf{n}\}$, то есть $\operatorname{Pol}\left(R_{4}\right)$.

5. $[\{\wedge, \neg, \otimes, \oplus\}]$ - это подкласс $P_{\{\mathbf{t}, \mathbf{b}, \mathbf{n}, \mathbf{f}\}}$, состоящий из всех $\leq_{k}$-монотонных функций, которые являются алгебраическими функциями как на $\{\mathbf{b}\}$, так и на $\{\mathbf{n}\}$, то есть $\operatorname{Pol}\left(R_{5}\right)$.

6. $[\{\wedge, \neg, \supset, \otimes, \oplus\}]-$ это подкласс $P_{\{\mathbf{t}, \mathbf{b}, \mathbf{n}, \mathbf{f}\}}$, состоящий из всех функций, которые являются алгебраическими функциями на $\{\mathbf{b}\}$, то есть $\operatorname{Pol}\left(R_{6}\right)$.

7. $\operatorname{Pol}\left(R_{7}\right)=[\{\wedge, \neg, \supset, \partial\}]-$ это подкласс $P_{\{\mathbf{t}, \mathbf{b}, \mathbf{n}, \mathbf{f}\}}$, состоящий из всех функций, которые являются алгебраическими функциями на $\{\mathbf{t}, \mathbf{f}\}$, то есть $\operatorname{Pol}\left(R_{7}\right)$.

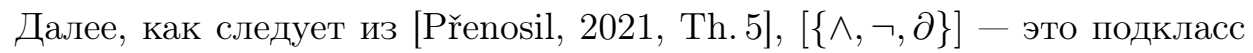
$P_{\{\mathbf{t}, \mathbf{b}, \mathbf{n}, \mathbf{f}\}}$, состоящий из всех функций, самодвойственных относительно $\partial$, то есть $\operatorname{Pol}\left(R_{8}\right)$. В оставшихся случаях $R_{9}-R_{14}$ имеет место следующее: $\operatorname{Pol}\left(R_{i}\right)=\left[\left\{\wedge, \vee, \neg, g_{i}\right\}\right]$, где $g_{i}$ определяется одной из приведенных ниже таблиц истинности. Достаточно убедиться, что для каждого $9 \leq i \leq 14$ функция $g_{i}$ сохраняет все предикаты из $R_{i}$ и не сохраняет никакие иные предикаты из множества $R_{F D E}$.

\begin{tabular}{c|cccc}
$g_{9}$ & $\mathbf{t}$ & $\mathbf{b}$ & $\mathbf{n}$ & $\mathbf{f}$ \\
\hline $\mathbf{t}$ & $\mathbf{n}$ & $\mathbf{n}$ & $\mathbf{b}$ & $\mathbf{f}$ \\
$\mathbf{b}$ & $\mathbf{f}$ & $\mathbf{f}$ & $\mathbf{f}$ & $\mathbf{f}$ \\
$\mathbf{n}$ & $\mathbf{f}$ & $\mathbf{f}$ & $\mathbf{n}$ & $\mathbf{f}$ \\
$\mathbf{f}$ & $\mathbf{f}$ & $\mathbf{f}$ & $\mathbf{f}$ & $\mathbf{f}$ \\
$g_{12}$ & $\mathbf{t}$ & $\mathbf{b}$ & $\mathbf{n}$ & $\mathbf{f}$ \\
\hline $\mathbf{t}$ & $\mathbf{f}$ & $\mathbf{n}$ & $\mathbf{b}$ & $\mathbf{f}$ \\
$\mathbf{b}$ & $\mathbf{f}$ & $\mathbf{f}$ & $\mathbf{f}$ & $\mathbf{f}$ \\
$\mathbf{n}$ & $\mathbf{f}$ & $\mathbf{f}$ & $\mathbf{n}$ & $\mathbf{f}$ \\
$\mathbf{f}$ & $\mathbf{f}$ & $\mathbf{f}$ & $\mathbf{f}$ & $\mathbf{f}$
\end{tabular}

\begin{tabular}{c|cccc}
$g_{10}$ & $\mathbf{t}$ & $\mathbf{b}$ & $\mathbf{n}$ & $\mathbf{f}$ \\
\hline $\mathbf{t}$ & $\mathbf{n}$ & $\mathbf{n}$ & $\mathbf{b}$ & $\mathbf{f}$ \\
$\mathbf{b}$ & $\mathbf{f}$ & $\mathbf{b}$ & $\mathbf{f}$ & $\mathbf{f}$ \\
$\mathbf{n}$ & $\mathbf{f}$ & $\mathbf{f}$ & $\mathbf{n}$ & $\mathbf{f}$ \\
$\mathbf{f}$ & $\mathbf{f}$ & $\mathbf{f}$ & $\mathbf{f}$ & $\mathbf{f}$ \\
& & & & \\
$g_{13}$ & $\mathbf{t}$ & $\mathbf{b}$ & $\mathbf{n}$ & $\mathbf{f}$ \\
\hline $\mathbf{t}$ & $\mathbf{f}$ & $\mathbf{n}$ & $\mathbf{b}$ & $\mathbf{f}$ \\
$\mathbf{b}$ & $\mathbf{f}$ & $\mathbf{b}$ & $\mathbf{f}$ & $\mathbf{f}$ \\
$\mathbf{n}$ & $\mathbf{f}$ & $\mathbf{f}$ & $\mathbf{f}$ & $\mathbf{f}$ \\
$\mathbf{f}$ & $\mathbf{f}$ & $\mathbf{f}$ & $\mathbf{f}$ & $\mathbf{f}$
\end{tabular}

\begin{tabular}{c|cccc}
$g_{11}$ & $\mathbf{t}$ & $\mathbf{b}$ & $\mathbf{n}$ & $\mathbf{f}$ \\
\hline $\mathbf{f}$ & $\mathbf{f}$ & $\mathbf{n}$ & $\mathbf{b}$ & $\mathbf{f}$ \\
$\mathbf{b}$ & $\mathbf{f}$ & $\mathbf{b}$ & $\mathbf{f}$ & $\mathbf{f}$ \\
$\mathbf{n}$ & $\mathbf{f}$ & $\mathbf{f}$ & $\mathbf{n}$ & $\mathbf{f}$ \\
$\mathbf{f}$ & $\mathbf{f}$ & $\mathbf{f}$ & $\mathbf{f}$ & $\mathbf{f}$ \\
& & & & \\
$g_{14}$ & $\mathbf{t}$ & $\mathbf{b}$ & $\mathbf{n}$ & $\mathbf{f}$ \\
\hline $\mathbf{f}$ & $\mathbf{f}$ & $\mathbf{n}$ & $\mathbf{b}$ & $\mathbf{f}$ \\
$\mathbf{b}$ & $\mathbf{f}$ & $\mathbf{b}$ & $\mathbf{f}$ & $\mathbf{f}$ \\
$\mathbf{n}$ & $\mathbf{f}$ & $\mathbf{n}$ & $\mathbf{n}$ & $\mathbf{f}$ \\
$\mathbf{f}$ & $\mathbf{f}$ & $\mathbf{f}$ & $\mathbf{f}$ & $\mathbf{f}$
\end{tabular}


Полученный результат позволяет не только перечислить замкнутые классы, которые служат предметом данной работы, но и упорядочить их по включению:

$$
\begin{gathered}
\operatorname{Pol}\left(\left\{\varrho_{1}^{1}, \varrho_{2}^{1}, \varrho_{3}^{1}, \varrho_{1}^{2}\right\}\right) \subseteq \operatorname{Pol}\left(\left\{\varrho_{1}^{1}, \varrho_{2}^{1}, \varrho_{3}^{1}\right\}\right) ; \operatorname{Pol}\left(\left\{\varrho_{1}^{1}, \varrho_{2}^{1}, \varrho_{3}^{1}, \varrho_{1}^{2}\right\}\right) \subseteq \operatorname{Pol}\left(\left\{\varrho_{3}^{1}, \varrho_{1}^{2}\right\}\right) ; \\
\operatorname{Pol}\left(\left\{\varrho_{1}^{1}, \varrho_{2}^{1}, \varrho_{3}^{1}\right\}\right) \subseteq \operatorname{Pol}\left(\left\{\varrho_{1}^{1}, \varrho_{2}^{1}\right\}\right) ; \operatorname{Pol}\left(\left\{\varrho_{1}^{1}, \varrho_{2}^{1}, \varrho_{3}^{1}\right\}\right) \subseteq \operatorname{Pol}\left(\left\{\varrho_{1}^{1}, \varrho_{3}^{1}\right\}\right) ; \\
\operatorname{Pol}\left(\left\{\varrho_{1}^{1}, \varrho_{2}^{1}, \varrho_{3}^{1}\right\}\right) \subseteq \operatorname{Pol}\left(\left\{\varrho_{2}^{1}, \varrho_{3}^{1}\right\}\right) ; \operatorname{Pol}\left(\left\{\varrho_{1}^{1}, \varrho_{2}^{1}, \varrho_{12}^{2}\right\}\right) \subseteq \operatorname{Pol}\left(\left\{\varrho_{1}^{1}, \varrho_{2}^{1}\right\}\right) ; \\
\operatorname{Pol}\left(\left\{\varrho_{1}^{1}, \varrho_{2}^{1}, \varrho_{12}^{2}\right\}\right) \subseteq \operatorname{Pol}\left(\left\{\varrho_{1}^{1}, \varrho_{12}^{2}\right\}\right) ; \operatorname{Pol}\left(\left\{\varrho_{1}^{1}, \varrho_{2}^{1}, \varrho_{12}^{2}\right\}\right) \subseteq \operatorname{Pol}\left(\left\{\varrho_{2}^{1}, \varrho_{12}^{2}\right\}\right) ; \\
\operatorname{Pol}\left(\left\{\varrho_{1}^{1}, \varrho_{2}^{1}\right\}\right) \subseteq \operatorname{Pol}\left(\left\{\varrho_{1}^{1}\right\}\right) ; \operatorname{Pol}\left(\left\{\varrho_{1}^{1}, \varrho_{2}^{1}\right\}\right) \subseteq \operatorname{Pol}\left(\left\{\varrho_{2}^{1}\right\}\right) ; \\
\operatorname{Pol}\left(\left\{\varrho_{1}^{1}, \varrho_{3}^{1}\right\}\right) \subseteq \operatorname{Pol}\left(\left\{\varrho_{1}^{1}\right\}\right) ; \operatorname{Pol}\left(\left\{\varrho_{1}^{1}, \varrho_{3}^{1}\right\}\right) \subseteq \operatorname{Pol}\left(\left\{\varrho_{3}^{1}\right\}\right) ; \\
\operatorname{Pol}\left(\left\{\varrho_{1}^{1}, \varrho_{12}^{2}\right\}\right) \subseteq \operatorname{Pol}\left(\left\{\varrho_{1}^{1}\right\}\right) ; \operatorname{Pol}\left(\left\{\varrho_{1}^{1}, \varrho_{12}^{2}\right\}\right) \subseteq \operatorname{Pol}\left(\left\{\varrho_{12}^{2}\right\}\right) ; \\
\operatorname{Pol}\left(\left\{\varrho_{2}^{1}, \varrho_{3}^{1}\right\}\right) \subseteq \operatorname{Pol}\left(\left\{\varrho_{2}^{1}\right\}\right) ; \operatorname{Pol}\left(\left\{\varrho_{2}^{1}, \varrho_{3}^{1}\right\}\right) \subseteq \operatorname{Pol}\left(\left\{\varrho_{3}^{1}\right\}\right) ; \\
\operatorname{Pol}\left(\left\{\varrho_{2}^{1}, \varrho_{12}^{2}\right\}\right) \subseteq \operatorname{Pol}\left(\left\{\varrho_{2}^{1}\right\}\right) ; \operatorname{Pol}\left(\left\{\varrho_{2}^{1}, \varrho_{12}^{2}\right\}\right) \subseteq \operatorname{Pol}\left(\left\{\varrho_{12}^{2}\right\}\right) ; \\
\operatorname{Pol}\left(\left\{\varrho_{3}^{1}\right\}\right) \subseteq \operatorname{Pol}(\{\varnothing\}) ; \operatorname{Pol}\left(\left\{\varrho_{12}^{2}\right\}\right) \subseteq \operatorname{Pol}(\{\varnothing\}) .
\end{gathered}
$$

Для наглядности изобразим отношения между классами на диаграмме (рис. 1). В качестве обозначений классов используем множества предикатов, которые они сохраняют.

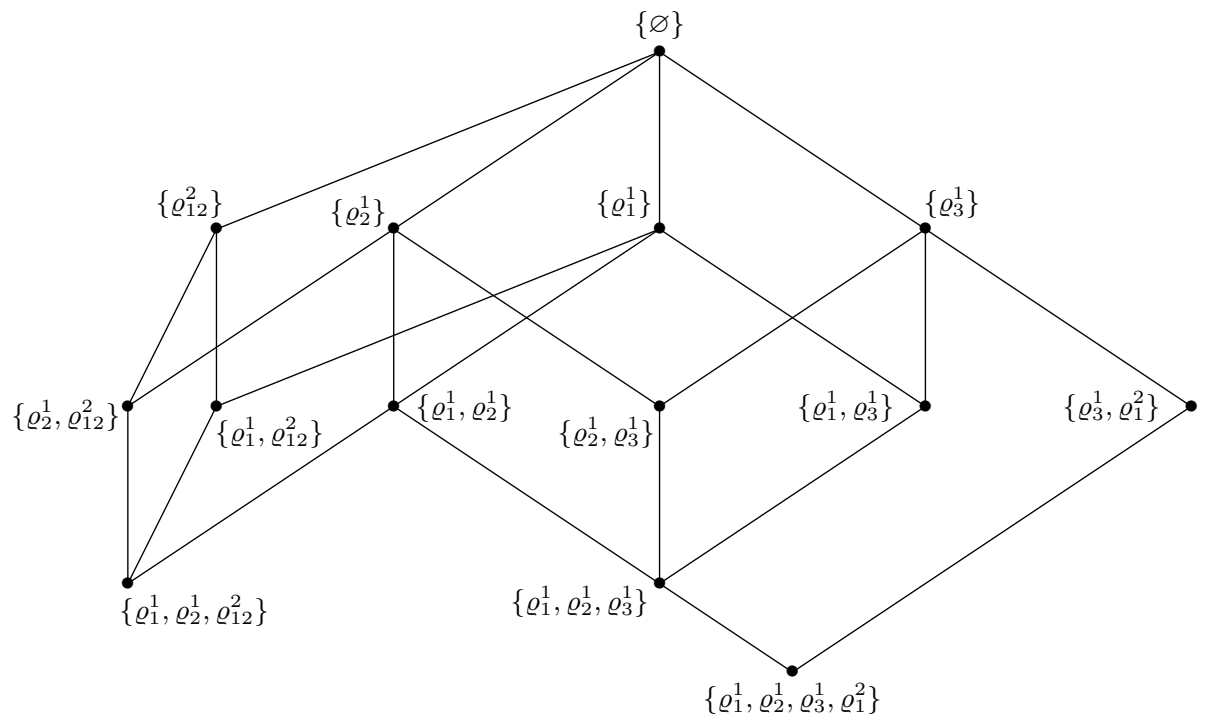

Рис. 1. Отношения между замкнутыми классами 


\section{Заключение}

Представленные результаты можно применить для анализа свойств четырехзначных расширений FDE, которые могут быть сформулированы через определимость в этих расширениях тех или иных операторов. Раскроем этот тезис на примере, установив связь между свойствами формальной противоречивости и неопределенности с определимостью классического отрицания в максимально параполных и максимально паранепротиворечивых четырехзначных расширениях FDE.

Пусть $\mathbf{F D E}^{\mathbf{x}}$ - четырехзначное языковое расширение FDE. Говорим, что $\mathbf{F D E}^{\mathbf{x}}$ - логика формальной противоречивости (LFI), если в $\mathbf{F D E}^{\mathbf{x}}$ имеет место $p \wedge \neg p \nvdash q ; p \wedge \circ p \nvdash q ; \neg p \wedge \circ p \nvdash q ; p \wedge \neg p \wedge \circ p \vdash q$ для некоторого унарного оператора о. Говорим, что $\mathbf{F D E}^{\mathbf{x}}$ - логика формальной неопределенности $(\mathbf{L F U})$, когда в $\mathbf{F D E}^{\mathbf{x}}$ для некоторого унарного оператора $\star$ верны следующие утверждения: $p \nvdash q \vee \neg q ; p \nvdash q \vee \star q ; p \nvdash \neg q \vee \star q$ $p \vdash q \vee \neg q \vee \star q$. В определениях понятий опираемся на [Marcos, 2005], адаптируя их для расширений FDE. Анализ данных свойств применительно к четырехзначным расширениям FDE можно найти в Omori, Sano, 2014 и Arieli, Avron, 2017.

Чтобы выполнялось условие $p \wedge \neg p \wedge$ о $\vdash q$, необходимо $\circ(\mathbf{b}) \in\{\mathbf{n}, \mathbf{f}\}$. При этом должны также выполняться условия $p \wedge \circ p \nvdash q$ и $\neg p \wedge \circ p \nvdash q$, поэтому о $(x) \in\{\mathbf{t}, \mathbf{b}\}$ для $x \in\{\mathbf{t}, \mathbf{f}\}$. Отсюда имеем следующее: если $\mathbf{F D E}^{\mathbf{x}}$ является $\mathbf{L F I}$, то $\left[F D E^{x}\right]$ не сохраняет ни $\varrho_{2}^{1}$, ни $\varrho_{12}^{2}$. Аналогичным образом $\star(\mathbf{n}) \in\{\mathbf{t}, \mathbf{b}\}$ и $\star(x) \in\{\mathbf{n}, \mathbf{f}\}$ для $x \in\{\mathbf{t}, \mathbf{f}\}$, поэтому если $\mathbf{F D E}^{\mathbf{x}}$ является $\mathbf{L F U}$, то $\left[F D E^{x}\right]$ не сохраняет ни $\varrho_{1}^{1}$, ни $\varrho_{12}^{2}$. Теперь пусть $\circ(x)=\mathbf{t}$, если $x \in\{\mathbf{t}, \mathbf{f}\}$ и $\circ(\mathbf{b})=\mathbf{n}, \circ(\mathbf{n})=\mathbf{b}$. Пусть также $\star(x)=\mathbf{f}$, если $x \in\{\mathbf{t}, \mathbf{f}\}$ и $\star(\mathbf{b})=\mathbf{n}, \star(\mathbf{n})=\mathbf{b}$. Тогда $[\{\circ, \star\}] \subseteq \operatorname{Pol}\left(\varrho_{1}^{2}\right)$. Как следствие, если $\mathbf{F D E}^{\mathbf{x}}-$ максимально паранепротиворечивое и максимально параполное языковое расширение $\mathbf{F D E}$, эта логика является одновременно логикой формальной противоречивости и логикой формальной неопределенности тогда и только тогда, когда $\left[F D E^{x}\right]=\operatorname{Pol}\left(R_{i}\right)$, где $i \in\{1,7,8\}$.

Теперь рассмотрим следующее свойство - определимость в расширении FDE классического отрицания. Анализу этого свойства отдельно посвящена работа Omori, Sano, 2015]. Говорим, что в $\mathbf{F D E}^{\mathbf{x}}$ определимо классическое отрицание, если в $\left[F D E^{x}\right]$ содержится такая функция $\sim$, что $\sim x \in\{\mathbf{t}, \mathbf{b}\} \Longleftrightarrow x \in\{\mathbf{n}, \mathbf{f}\}$. Если выполнено это условие, то $\sim$ не сохраняет ни $\varrho_{1}^{1}$, ни $\varrho_{2}^{1}$, ни $\varrho_{12}^{2}$. Пусть $\sim(\mathbf{t})=\mathbf{f}, \sim(\mathbf{b})=\mathbf{n}, \sim(\mathbf{n})=\mathbf{b}$, $\sim(\mathbf{f})=\mathbf{t}$. В этом случае $\sim$ сохраняет $\varrho_{1}^{2}$. Как следствие, если $\mathbf{F D E}^{\mathbf{x}}-$ максимально паранепротиворечивое и максимально параполное языковое расширение FDE, в этой логике определимо классическое отрицание то- 
гда и только тогда, когда она является одновременно логикой формальной противоречивости и логикой формальной неопределенности.

Мы показали, как замкнутые классы могут быть поставлены в соответствие тем или иным свойствам расширений FDE. Конечно, применимость этого метода не исчерпывается приведенным примером. Например, в Рřenosil, 2021 аналогичный подход применяется для анализа расширений FDE с точки зрения абстрактной алгебраической логики - замкнутым классам функций сопоставляются свойства «быть протоалгебраической логикой», «быть алгебраизируемой логикой», «быть импликативной логикой» и др.

Еще одна область применения результатов статьи касается полученного нами множества предикатов $R_{F D E}$. Оно позволяет сравнить выразительные возможности двух произвольных четырехзначных языковых расширений FDE. Для этого достаточно установить, какое подмножество $R_{F D E}$ сохраняют одновременно все элементарные операции матрицы первого из сравниваемых расширений, а какое - второго.

Завершая статью, остановимся на возможных направлениях дальнейших исследований. В статьях Shramko et al., 2017, Shramko et al., 2019 рассматриваются родственные FDE логики ETL и NFL, характеристические матрицы которых отличаются от $\mathcal{M}_{\text {FDE }}$ только классами выделенных значений. В то время как $D=\{\mathbf{t}, \mathbf{b}\}$ в $\mathcal{M}_{\mathbf{F D E}}, D=\{\mathbf{t}\}$ в $\mathcal{M}_{\mathbf{E T L}}$ и $D=\{\mathbf{t}, \mathbf{b}, \mathbf{n}\}$ в $\mathcal{M}_{\mathbf{N F L}}$. Логика ETL параполна, но не паранепротиворечива. Логика NFL паранепротиворечива, но не параполна. Представляют интерес критерии максимальной паранепротиворечивости (или параполноты) в данных логиках, а также замкнутые классы, порождаемые операциями их максимально паранепротиворечивых (или параполных) четырехзначных языковых расширений. Кроме того, актуальной представляется задача построения полной решетки замкнутых надклассов $[F D E]$, а не только ее отдельных фрагментов.

\section{Литература}

Жук, 2011 - Жук Д.Н. Предикатный метод построения решетки Поста // Дискретная математика. 2011. Т. 23. № 2. С. 115-128.

Жук, 2018 - Жук Д.Н. От двузначной к $k$-значной логике // Интеллектуальные системы. Теория и приложения. 2018. Т. 22. Вып. 1. С. 131-149.

Нагорный, 2013 - Нагорный А.C. О пересечениях и объединениях предполных классов многозначной логики: дис. . . . канд. физ.-мат. наук: 01.01.09. М., 2013. $162 \mathrm{c}$.

Adams, Dziobiak, 1994 - Adams M.E, Dziobiak W. Lattices of quasivarieties of 3-element algebras // Journal of Algebra. 1994. Vol. 166. No. 1. P. 181-210. 
Avron, 1999 - Avron A. On the expressive power of three-valued and four-valued languages // Journal of Logic and Computation. 1999. Vol. 9. No. 6. P. 977-994.

Arieli, Avron, 1998 - Arieli O., Avron A. The value of the four values // Artificial Intelligence. 1998. Vol. 102. No. 1. P. 97-141.

Arieli, Avron, 2017 - Arieli O., Avron A. Four-valued paradefinite logics // Studia Logica. 2017. Vol. 105. No. 6. P. 1087-1122.

Arieli et al., 2011 - Arieli O., Avron A., Zamansky A. Maximal and premaximal paraconsistency in the framework of three-valued semantics // Studia Logica. 2011. Vol. 97. No. 1. P. 31-60.

Baker, Pixley, 1975 - Baker K.A., Pixley A.F. Polynomial interpolation and the Chines Remainder Theorem for algebraic systems // Mathematische Zeitschrift. 1975. Vol. 143. No. 2. P. 165-174.

Belnap, 1977 - Belnap N.D. How a computer should think // Contemporary Aspects of Philosophy / Ed. by G. Ryle. Oriel Press, 1977. P. 30-56.

Da Costa, 1974 - Da Costa N.C.A. On the theory of inconsistent formal systems // Notre Dame Journal of Formal Logic. 1974. Vol. 15. No. 4. P. 497-510.

De, Omori, 2015 - De M., Omori H. Classical Negation and Expansions of BelnapDunn Logic // Studia Logica. 2015. Vol. 103. No. 5. P. 825-851.

Karpenko, 2017 - Karpenko A.S. Four-valued logics BD and DM4: Expansions Bulletin of the Section of Logic. 2017. Vol. 46. No. 1-2. P. 33-45.

Lau, 2006 - Lau D. Function algebras on finite sets: Basic course on many-valued logic and clone theory. Springer: Science \& Business Media, 2006. 670 c.

Łoś, 1949 - Łoś J. O matrycach logicznych // Prace Wroclawskiego Towarzystwa Naukowego. Seria B. 1949. No. 19. P. 1-141.

Marcos, 2005 - Marcos J. Nearly every normal modal logic is paranormal // Logique et Analyse. 2005. Vol. 48. No. 189-192. P. 279-300.

Omori, Sano, 2014 - Omori H., Sano K. Da Costa meets Belnap and Nelson // Recent Trends in Philosophical Logic / Ed. by R. Ciuni, H. Wansing, C. Willkommen. Springer, 2014. P. 145-166.

Omori, Sano, 2015 - Omori H., Sano K. Generalizing functional completeness in Belnap-Dunn logic // Studia Logica. 2015. Vol. 103. No. 5. P. 883-917.

Omori, Wansing, 2017 - Omori H., Wansing H. 40 years of FDE: an introductory overview // Studia Logica. 2017. Vol. 105. No. 6. P. 1021-1049.

Petrukhin, Shangin, 2019 - Petrukhin Ya.I., Shangin V.O. Correspondence analysis and automated proof-searching for first degree entailment // European Journal of Mathematics. 2019. P. 1-44.

Přenosil, 2021 - Přenosil A. De Morgan clones and four-valued logics // Algebra universalis. 2021. Vol. 82. No. 2. P. 1-42.

Priest, 2008 - Priest G. An introduction to non-classical logic: From if to is. Cambridge University Press, 2008. 613 p. 
Pynko, 1999 - Pynko A. Functional Completeness and Axiomatizability within Belnap's Four-Valued Logic and its Expansions // Journal of Applied NonClassical Logics. 1999. Vol. 9. No. 1. P. 61-105.

Shapiro, 2017 - Shapiro L. LP, K3 and FDE as Substructural Logics // The Logica Yearbook 2016 / Ed. by P. Arazim and T. Lavička. London: College Publications, 2017. P. 257-272.

Shoesmith, Smiley, 1978 - Shoesmith D.J., Smiley T.J. Multiple-conclusion logic. CUP Archive, 1978. 396 p.

Shramko et al., 2017 - Shramko Y., Zaitsev D., Belikov A. First-Degree Entailment and its Relatives // Studia Logica. 2017. Vol. 105. P. 1291-1317.

Shramko et al., 2019 - Shramko Y., Zaitsev D., Belikov A. The FMLA-FMLA Axiomatizations of the Exactly True and Non-falsity Logics and Some of Their Cousins // Journal of Philosophical Logic. 2019. Vol. 48. P. 787-808.

Tomova, 2021 - Tomova N. A Semi-lattice of Four-valued Literal-paraconsistentparacomplete Logics // Bulletin of the Section of Logic. 2021. Vol. 50. No. 1. P. 35-53. 
LEONID YU. DEVYATKIN

\title{
On the expressive power of maximally paraconsistent and maximally paracomplete expansions of FDE
}

\author{
Leonid Yu. Devyatkin \\ Institute of Philosophy, Russian Academy of Sciences, \\ 12/1 Goncharnaya Str., Moscow, 109240, Russian Federation. \\ E-mail: deviatkin@iph.ras.ru
}

\begin{abstract}
The paper is devoted to the closed classes of functions of four-valued logics which can be generated by the primitive operations of characteristic matrices for the expansions of FDE that are simultaneously maximally paraconsistent and paracomplete.
\end{abstract}

We begin with presenting the necessary and sufficient conditions which four-valued expansions of FDE must satisfy in order to be maximally paraconsistent and paracomplete. In both cases, the criteria of maximality are linked to operations of a certain kind being present in a matrix of an expansion in question, which prevent it from being a sublogic of a three-valued expansion of Asenjo-Priest's logic $\mathbf{L P}$ - in the paraconsistent case, or Kleene's logic $\mathbf{K}_{\mathbf{3}}-$ in the paracomplete case.

Further, relying on the Baker-Pixley theorem, we describe a set of 5 unary and 20 binary predicates such that any closed class of functions generated by the operations of a four-valued characteristic matrix characterizing an expansion of FDE is a class of functions that preserve one of its subsets. This yields a simple algorithm for comparing the expressive power of any arbitrary four-valued expansion of FDE.

Moreover, taking into account that the given set of predicates includes all predicates describing precomplete classes of functions of four-valued logic which are preserved by the operations of the characteristic matrix for FDE, we provide criteria of functional completeness and precompleteness for the operations of any four-valued matrix which characterizes an expansion of FDE.

Finally, by utilizing the conditions of maximal paraconsistency and paracompleteness, as well as the list of predicates for expansions of FDE, given in the paper, we identify all 14 sets of predicates describing the closed classes of functions which can be generated by systems of primitive operations of four-valued characteristic matrices for such expansions of FDE that are at the same time maximally paraconsistent and maximally paracomplete. This allows us to not only list all closed classes corresponding to the maximally paraconsistent and paracomplete four-valued expansions of FDE, but also to order them with respect to inclusion.

Keywords: four-valued logics, paraconsistency, paracompleteness, closed classes of functions, closed classes of predicates

For citation: Devyatkin L.Yu. "O vyrazitel'nykh vozmozhnostyakh maksimal'no paraneprotivorechivykh i maksimal'no parapolnykh chetyrekhznachnykh rasshirenii FDE " 
[On the expressive power of maximally paraconsistent and maximally paracomplete expansions of FDE], Logicheskie Issledovaniya / Logical Investigations, 2021, Vol. 27, No. 2, pp. 6692. DOI: 10.21146/2074-1472-2021-27-2-66-92 (In Russian)

\section{References}

Zhuk, 2011 - Zhuk, D.N. "Predikatnyi metod postroeniya reshetki Posta" [The predicate method for constructing Post's lattice], Diskretnaya Matematika, 2011, Vol. 23, No. 2, pp. 115-128. (In Russian)

Zhuk, 2018 - Zhuk, D.N. "Ot dvuznachnoi k $k$-znachnoi logike" [From two-valued to $k$-valued, logic], Intellektual'nye sistemy. Teoriya $i$ prilozheniya, 2018, Vol. 22, Is. 1, pp. 131-149. (In Russian)

Nagorny, 2013 - Nagorny, A.S, O peresecheniyakh i ob'edineniyakh predpolnykh klassov mnogoznachnoi logiki [On meets and joins of precomplete classes of manyvalued logic], Moscow, 2013. 162 pp. (In Russian)

Adams, Dziobiak, 1994 - Adams M.E, Dziobiak W. "Lattices of quasivarieties of 3-element algebras", Journal of Algebra, 1994, Vol. 166, No. 1, pp. 181-210.

Avron, 1999 - Avron, A. "On the expressive power of three-valued and four-valued languages", Journal of Logic and Computation, 1999, Vol. 9, No. 6, pp. 977-994.

Arieli, Avron, 1998 - Arieli, O., Avron, A. "The value of the four values", Artificial Intelligence, 1998, Vol. 102, No. 1, pp. 97-141.

Arieli, Avron, 2017 - Arieli, O., Avron, A. "Four-valued paradefinite logics", Studia Logica, 2017, Vol. 105, No. 6, pp. 1087-1122.

Arieli et al., 2011 - Arieli, O., Avron, A., Zamansky, A. "Maximal and premaximal paraconsistency in the framework of three-valued semantics", Studia Logica, 2011, Vol. 97, No. 1, pp. 31-60.

Baker, Pixley, 1975 - Baker, K.A., Pixley, A.F "Polynomial interpolation and the Chinese Remainder Theorem for algebraic systems", Mathematische Zeitschrift, 1975, Vol. 143, No. 2, pp. 165-174.

Belnap, 1977 - Belnap, N. "A useful four-valued logic", Modern Uses of MultipleValued Logic, ed. by J.M. Dunn, G. Epstein. Boston: D. Reidel Publishing Co., 1977, pp. 8-37.

Da Costa, 1974 - Da Costa, N.C.A. "On the theory of inconsistent formal systems", Notre Dame Journal of Formal Logic, 1974, Vol. 15, No. 4, pp. 497-510.

De, Omori, 2015 - De, M., Omori, H., "Classical Negation and Expansions of BelnapDunn Logic", Studia Logica, 2015, Vol. 103, No. 5, pp. 825-851.

Karpenko, 2017 - Karpenko, A.S., "Four-valued logics BD and DM4: Expansions", Bulletin of the Section of Logic, 2017, Vol. 46, No. 1-2, pp. 33-45.

Lau, 2006 - Lau, D. Function algebras on finite sets: Basic course on many-valued logic and clone theory. Springer: Science \& Business Media, 2006. 670 pp.

Łoś, 1949 - Łoś, J. "O matrycach logicznych", Prace Wroclawskiego Towarzystwa Naukowego. Seria B, 1949, No. 19, pp. 1-141. (In Polish) 
Marcos, 2005 - Marcos, J. "Nearly every normal modal logic is paranormal", Logique et Analyse, 2005, Vol. 48, No. 189-192, pp. 279-300.

Omori, Sano, 2014 - Omori, H., Sano, K. "da Costa meets Belnap and Nelson", Recent Trends in Philosophical Logic, ed. by R. Ciuni, H. Wansing, C. Willkommen. Springer, 2014, pp. 145-166.

Omori, Sano, 2015 - Omori, H., Sano, K. "Generalizing functional completeness in Belnap-Dunn logic", Studia Logica, 2015, Vol. 103, No. 5, pp. 883-917.

Omori, Wansing, 2017 - Omori, H., Wansing, H. "40 years of FDE: an introductory overview", Studia Logica, 2017, Vol. 105, No. 6, pp. 1021-1049.

Petrukhin, Shangin, 2019 - Petrukhin, Ya.I., Shangin, V.O. "Correspondence analysis and automated proof-searching for first degree entailment", European Journal of Mathematics, 2019, pp. 1-44.

Přenosil, 2021 - Přenosil, A. "De Morgan clones and four-valued logics", Algebra universalis, 2021, Vol. 82, No. 2, pp. 1-42.

Priest, 2008 - Priest, G. An introduction to non-classical logic: From if to is. Cambridge; New York: Cambridge University Press, 2008. 613 pp.

Pynko, 1999 - Pynko, A. "Functional Completeness and Axiomatizability within Belnap's Four-Valued Logic and its Expansions", Journal of Applied Non-Classical Logics, 1999, Vol. 9, No. 1, pp. 61-105.

Shapiro, 2017 - Shapiro, L. "LP, K3 and FDE as Substructural Logics", The Logica Yearbook 2016, ed. by P. Arazim and T. Lavička. London: College Publications, 2017, pp. 257-272.

Shoesmith, Smiley, 1978 - Shoesmith, D.J., Smiley, T.J. Multiple-conclusion logic, CUP Archive, 1978. 396 pp.

Shramko et al., 2017 - Shramko, Y., Zaitsev, D., Belikov, A. "First-Degree Entailment and its Relatives", Studia Logica, 2017, Vol. 105, pp. 1291-1317.

Shramko et al., 2019 - Shramko, Y., Zaitsev, D., Belikov, A. "The FMLA-FMLA Axiomatizations of the Exactly True and Non-falsity Logics and Some of Their Cousins", Journal of Philosophical Logic, 2019, Vol. 48, pp. 787-808.

Tomova, 2021 - Tomova N. "A Semi-lattice of Four-valued Literal-paraconsistentparacomplete Logics", Bulletin of the Section of Logic, 2021, Vol. 50, No. 1, pp. 35-53. 